\title{
Delta - Open Sets And Delta - Continuous Functions
}

\author{
Raja Mohammad Latif \\ Department of Mathematics and Natural Sciences \\ Prince Mohammad Bin Fahd University \\ P.O. Box 1664 Al - Khobar 31952 \\ Saudi Arabia
}

\begin{abstract}
In 1968 Velicko [30] introduced the concepts of $\delta$-closure and $\delta$-interior operations. We introduce and study properties of $\delta$-derived, $\delta$-border, $\delta$-frontier and $\delta$-exterior of a set using the concept of $\delta$-open sets. We also introduce some new classes of topological spaces in terms of the concept of $\delta-D-$ sets and investigate some of their fundamental properties. Moreover, we investigate and study some further properties of the well-known notions of $\delta$-closure and $\delta$-interior of a set in a topological space. We also introduce $\delta-R_{0}$ space and study its characteristics. We also introduce $\delta-R_{0}$ space and study its characteristics. We introduce $\delta$-irresolute, $\delta$-closed, $\quad$ pre- $\delta$-open and pre- $\delta$-closed mappings and investigate
\end{abstract} properties and characterizations of these new types of mappings and also explore further properties of the well-known notions of $\delta$-continuous and $\delta$-open mappings.

\section{Keywords-Pure Mathematics, Topology}

\section{INTRODUCTION AND PRELIMINARIES}

Velicko [30] introduced the notion of $\delta$-closure, $\quad$ and $\delta$-int erior operations. Throughout this paper, $(X, \tau)$ (simply $X)$ always mean topological space on which no separation axioms are assumed unless explicitly stated. Let $S$ be a subset of $X$. The closure (resp., interior) of $S$ will be denoted by $\mathrm{Cl}(S)$, $(\operatorname{resp} ., \operatorname{Int}(S))$. A subset $S$ of $X$ is called a semi-open set [21] if $S \subseteq C l[\operatorname{Int}(S)]$. The complement of a semi-open set is called a semiclosed set. The intersection of all semi-closed sets containing $A$ is called the semi-closure of $A$ and is denoted by $\operatorname{sCl}(A)$. The family of all semi-open sets in a topological space $(X, \tau)$ will be denoted by $S O(X, \tau)$. A subset $M(X)$ of a space $X$ called a semi-neighborhood of a point $x \in X$ if there exists a semi-open set $S$ such that $x \in S \subseteq M(x)$. In [19] Latif introduced the notion of semi-convergence of filters and investigated some characterizations related to semi-open continuous function. A point $x \in X$ is called the $\delta$-cluster point of $A \subseteq X$ if $A$ I $\operatorname{Int}[C l(U)] \neq \phi$ for every open set $U$ of $X$ containing $x$. The set of all $\delta$-cluster points of $A$ is called the $\delta$-closure of $A$, denoted by $C l_{\delta}(A)$. A subset $A \subseteq X$ is called $\delta$-closed if $A=C l_{\delta}(A)$. The complement of a $\delta$-closed set is called $\delta$-open. The collection of all $\delta$-open sets in a topological space $(X, \tau)$ forms a topology $\tau_{\delta}$ on $X$, called the semigeneralization topology of $\tau$, weaker than $\tau$ and the class of all regular open sets in $\tau$ forms an open basis for $\left(X, \tau_{\delta}\right)$. In this paper, we introduce and study properties of $\delta$-derived, $\delta$-border, $\delta$-frontier and $\delta$-exterior of a set using the concept of $\delta$-open and study also other properties of the well-known notions of $\delta$-closure and $\delta$-interior. The notion of $\theta$-open subsets, $\theta$-closed subsets and $\theta$-closure were introduced by Velicko [30] for the purpose of studying the important class of 
$H$-closed spaces in terms of arbitrary filterbases. A point $x \in X$ is called a $\theta$-adherent point of $A$ [7], if $A \mathrm{I} C l(V) \neq \phi$ for every open set $V$ containing $x$. The set of all $\theta$-adherent points of $A$ is called the $\theta$-closure of $A$ and is denoted by $C l_{\theta}(A)$. A subset $A$ of $X$ is called $\theta$-closed if $A=C l_{\theta}(A)$. Dontchev and Maki [[7], Lemma 3.9] have shown that if $A$ and $B$ are subsets of a space $(X, \tau)$, then $C l_{\theta}(A \mathrm{U} B)=C l_{\theta}(A) \mathrm{U} C l_{\theta}(B) \quad$ and $C l_{\theta}(A \mathrm{I} B)=C l_{\theta}(A) \mathrm{I} C l_{\theta}(B)$. Note also that the $\theta$-closure of a given set need not be a $\theta$-closed set. But it is always closed. The complement of a $\theta$-closed set is called a $\theta$-open set. The $\theta$-interior of set $A$ in $X$, written $\operatorname{Int}_{\theta}(A)$, consists of those points $x$ of $A$ such that for some open set $U$ containing $x$, $C l(U) \subseteq A$. A set $A$ is $\theta$-open if and only if $A=\operatorname{Int}_{\theta}(A), \quad$ or equivalently, $\quad X-A$ is $\theta$-closed. The collection of all $\theta$-open sets in a topological space $(X, \tau)$ forms a topology $\tau_{\theta}$ on $\boldsymbol{X}$, weaker than $\tau$. We observe that for any topological space $(X, \tau)$, the relation $\tau_{\theta} \subseteq \tau_{\delta} \subseteq \tau$ always holds. We also have $A \subseteq C l(A) \subseteq C l_{\delta}(A) \subseteq C l_{\theta}(A)$, for any subset $A$ of $X$.

\section{BASIC PROPERTIES AND APPLICATIONS OF}

\section{DELTA OPEN SETS}

Definition 1.1. Let $A$ be a subset of a topological space $(X, \tau)$. A point $x \in A$ is said to be a $\delta$-limit point of $A$ if for each $\delta$-open set $U$ containing $x, U$ I $(A-\{x\}) \neq \phi$.

The set of all $\delta$-limit points of $A$ is called the $\delta$-derived set of $A$ and is denoted by $D_{\delta}(A)$.
Theorem 1.2. For subsets $A, B$ of a space $X$, the following statements hold:

(1) $D(A) \subseteq D_{\delta}(A)$, where $D(A)$ is the derived set of $A$;

(2) If $A \subseteq B$, then $D_{\delta}(A) \subseteq D_{\delta}(B)$;

(3) $D_{\delta}(A) \mathrm{U} D_{\delta}(B)=D_{\delta}(A \mathrm{U} B)$ and $D_{\delta}(A \mathrm{I} B) \subseteq D_{\delta}(A) \mathrm{I} D_{\delta}(B)$;

(4) $\left[D_{\delta}\left(D_{\delta}(A)\right)-A\right] \subseteq D_{\delta}(A)$;

(5) $D_{\delta}\left[A \cup D_{\delta}(A)\right] \subseteq\left[A \cup D_{\delta}(A)\right]$.

Proof. (1) It suffices to observe that every $\delta$-open set is an open set.

(2) Obvious.

$$
D_{\delta}(A) \mathrm{U} D_{\delta}(B)=D_{\delta}(A \cup B) \quad \text { is } \quad \text { a }
$$
modification of the standard proof for $\mathrm{D}$, where open sets are replaced by $\delta$-open sets. $D_{\delta}(A \mathrm{I} B) \subseteq D_{\delta}(A) \mathrm{I} D_{\delta}(B)$ follows by (2).

(4) If $x \in\left[D_{\delta}\left(D_{\delta}(A)\right)-A\right]$ and $U$ is a $\delta$-open set containing $x$, then $U$ I $\left[D_{\delta}(A)-\{x\}\right] \neq \phi$. Let $y \in U$ I $\left[D_{\delta}(A)-\{x\}\right]$. Then, since $y \in D_{\delta}(A)$ and $y \in U$, so $U \mathrm{I}[A-\{y\}] \neq \phi$. Let $z \in U \mathrm{I}[A-\{y\}]$. Then, $z \neq x$ for $z \in A$ and $x \notin A$. Hence, $U \mathrm{I}[A-\{x\}] \neq \phi$. Therefore, $x \in D_{\delta}(A)$.

(5) Let $x \in D_{\delta}\left[A \cup D_{\delta}(A)\right]$. If $x \in A$, the result is obvious. So, let $x \in\left[D_{\delta}\left(A \cup D_{\delta}(A)\right)-A\right]$, then, for $\delta$-open set $U$ containing $x$, $U \mathrm{I}\left[A \mathrm{U} D_{\delta}(A)-\{x\}\right] \neq \phi . \quad$ Thus, $U \mathrm{I}[A-\{x\}] \neq \phi$ or $U \mathrm{I}\left[D_{\delta}(A)-\{x\}\right] \neq \phi$. Now, it follows similarly from (4) that $U$ I $[A-\{x\}] \neq \phi . \quad$ Hence, $\quad x \in D_{\delta}(A)$. 
Therefore, in any case,
$D_{\delta}\left[A \cup D_{\delta}(A)\right] \subseteq\left[A \cup D_{\delta}(A)\right]$. Theorem 1.3. For any subset $A$ of a space $X$, $C l_{\delta}(A)=A \cup D_{\delta}(A)$.

Proof. Since $\quad D_{\delta}(A) \subseteq C l_{\delta}(A)$, $A \mathrm{U} D_{\delta}(A) \subseteq C l_{\delta}(A)$. On the other hand, let $x \in C l_{\delta}(A)$. If $x \in A$, then the proof is complete. If $x \notin A$, each $\delta$-open set $U$ containing $x$ intersects $A$ at a point distinct from $x$; so $x \in D_{\delta}(A)$. Thus, $C l_{\delta}(A) \subseteq\left[A \cup D_{\delta}(A)\right]$, which completes the proof.

Corollary 1.4. A subset $A$ is $\delta$-closed if and only if it contains the set of its $\delta$-limit it points.

Def inition 1.5. A point $x \in X$ is said to be a $\delta$-interior point of $A$ if there exists a $\delta$-open set $U$ containing $x$ such that $U \subseteq A$. The set of all $\delta$-interior points of $A$ is said to be $\delta$-interior of $A$ and is denoted by $\operatorname{Int}_{\delta}(A)$.

Theorem 1.6. For subsets $A, B$ of a space $X$, the following statements are true:

(1) $\operatorname{Int}_{\delta}(A)$ is the largest $\delta$-open set contained in $A$;

(2) $A$ is $\delta$-open if and only if $A=\operatorname{Int}_{\delta}(A)$;

(3) $\operatorname{Int}_{\delta}\left[\operatorname{Int}_{\delta}(A)\right]=\operatorname{Int}_{\delta}(A)$;

(4) $\operatorname{Int}_{\delta}(A)=\left[A-D_{\delta}(X-A)\right]$;

(5) $\left[X-\operatorname{Int}_{\delta}(A)\right]=C l_{\delta}(X-A)$;

(6) $\left[X-C l_{\delta}(A)\right]=\operatorname{Int}_{\delta}(X-A)$;

(7) $A \subseteq B$, then $\operatorname{Int}_{\delta}(A) \subseteq \operatorname{Int}_{\delta}(B)$.
(8) $\operatorname{Int}_{\delta}(A) \mathrm{U} \operatorname{Int}_{\delta}(B) \subseteq \operatorname{Int}_{\delta}(A \mathrm{U} B)$;

(9) $\operatorname{Int}_{\delta}(A \mathrm{I} B)=\operatorname{Int}_{\delta}(A) \mathrm{I} \operatorname{Int}_{\delta}(B)$;

Proof. (4) If $x \in\left[A-D_{\delta}(X-A)\right]$, then $x \notin D_{\delta}(X-A)$ and so there exists a $\delta$-open set $U$ containing $x$ such that $U \mathrm{I}(X-A)=\phi$. Then, $x \in U \subseteq A$ and hence $x \in \operatorname{Int}_{\delta}(A)$, that is, $\left[A-D_{\delta}(X-A)\right] \subseteq \operatorname{Int}_{\delta}(A)$. On the other hand, if $x \in \operatorname{Int}_{\delta}(A)$, then $x \notin D_{\delta}(X-A)$ since $\operatorname{Int}_{\delta}(A)$ is $\delta$-open and $\left[\operatorname{Int}_{\delta}(A) \mathrm{I}(X-A)\right]=\phi$. Hence, $\operatorname{Int}_{\delta}(A)=\left[A-D_{\delta}(X-A)\right]$.

(5) $X-\operatorname{Int}_{\delta}(A)=X-\left[A-D_{\delta}(X-A)\right]=$ $(X-A) \cup D_{\delta}(X-A)=C l_{\delta}(X-A)$

Def inition 1.7. $B d_{\delta}(A)=A-\operatorname{Int}_{\delta}(A)$ is said to be the $\delta$-border of $A$.

Theorem 1.8. For a subset $A$ of a space $X$, the following statements hold:

(1) $B d(A) \subseteq B d_{\delta}(A)$ where $B d(A)$ denotes the border of $A$;

(2) $A=\operatorname{Int}_{\delta}(A) \mathrm{U} B d_{\delta}(A)$;

(3) $\operatorname{Int}_{\delta}(A) \mathrm{I} B d_{\delta}(A)=\phi$;

(4) $A$ is a $\delta$-open set if and only if $B d_{\delta}(A)=\phi$;

(5) $B d_{\delta}\left[\operatorname{Int}_{\delta}(A)\right]=\phi$;

(6) $\operatorname{Int}_{\delta}\left[B d_{\delta}(A)\right]=\phi$;

(7) $B d_{\delta}\left[B d_{\delta}(A)\right]=B d_{\delta}(A)$;

(8) $B d_{\delta}(A)=A \mathrm{I}\left[C l_{\delta}(X-A)\right]$;

(9) $B d_{\delta}(A)=D_{\delta}(X-A)$. 
Proof. (6) If $\quad x \in \operatorname{Int}_{\delta}\left[B d_{\delta}(A)\right], \quad$ then $x \in B d_{\delta}(A)$. On the other hand, since $B d_{\delta}(A) \subseteq A, x \in \operatorname{Int}_{\delta}\left[B d_{\delta}(A)\right] \subseteq \operatorname{Int}_{\delta}(A)$. Hence, $x \in \operatorname{Int}_{\delta}(A) \mathrm{I} B d_{\delta}(A)$, which contradicts (3). Thus, $\operatorname{Int}_{\delta}\left[B d_{\delta}(A)\right]=\phi$.

(8) $B d_{\delta}(A)=A-\operatorname{Int}_{\delta}(A)=A-\left[X-C l_{\delta}(X-A)\right]=$ $A \mathrm{I} C l_{\delta}(X-A)$.

(9) $B d_{\delta}(A)=A-\operatorname{Int}_{\delta}(A)=A-\left[A-D_{\delta}(X-A)\right]=$ $D_{\delta}(X-A)$.

Def inition 1.9. $F r_{\delta}(A)=C l_{\delta}(A)-\operatorname{Int}_{\delta}(A)$ is said to be the $\delta$-frontier of $A$.

Theorem 1.10. For a subset $A$ of a space $X$, the following statements hold:

(1) $\operatorname{Fr}(A) \subseteq F r_{\delta}(A)$ where $\operatorname{Fr}(A)$ denotes the frontier of $A$;

(2) $C l_{\delta}(A)=\operatorname{Int}_{\delta}(A) U F r_{\delta}(A)$;

(3) $\operatorname{Int}_{\delta}(A) \operatorname{I} F r_{\delta}(A)=\phi$;

(4) $B d_{\delta}(A) \subseteq F r_{\delta}(A)$;

(5) $F r_{\delta}(A)=B d_{\delta}(A) \cup D_{\delta}(A)$;

(6) $A$ is a $\delta$-open set if and only if $\operatorname{Fr}_{\delta}(A)=D_{\delta}(A)$;

(7) $F r_{\delta}(A)=C l_{\delta}(A) \mathrm{I} C l_{\delta}(X-A)$;

(8) $F r_{\delta}(A)=F r_{\delta}(X-A)$;

(9) $F r_{\delta}(A)$ is $\delta$-closed;

(10) $F r_{\delta}\left[F r_{\delta}(A)\right] \subseteq F r_{\delta}(A)$;

(11) $\operatorname{Fr}_{\delta}\left[\operatorname{Int}_{\delta}(A)\right] \subseteq F r_{\delta}(A)$;
(12) $F r_{\delta}\left[C l_{\delta}(A)\right] \subseteq F r_{\delta}(A)$;

(13) $\operatorname{Int}_{\delta}(A)=A-F r_{\delta}(A)$.

Proof. (2) $I n t_{\delta}(A) U F r_{\delta}(A)=$ $\operatorname{Int}_{\delta}(A) \mathrm{U}\left[C l_{\delta}(A)-\operatorname{Int}_{\delta}(A)\right]=C l_{\delta}(A)$

(3) $\operatorname{Int}_{\delta}(A) \operatorname{I} F r_{\delta}(A)=\operatorname{Int}_{\delta}(A) \mathrm{I}\left[C l_{\delta}(A)-\operatorname{Int}_{\delta}(A)\right]=\phi$.

(5) Since $\operatorname{Int}_{\delta}(A) U F r_{\delta}(A)=$ $\operatorname{Int}_{\delta}(A) \mathrm{U} B d_{\delta}(A) \mathrm{U} D_{\delta}(A), F r_{\delta}(A)=B d_{\delta}(A) \mathrm{U} D_{\delta}(A)$

(7) $F r_{\delta}(A)=C l_{\delta}(A)-\operatorname{Int}_{\delta}(A)=C l_{\delta}(A) \mathrm{I} C l_{\delta}(X-A)$.

(9) $C l_{\delta}\left[F r_{\delta}(A)\right]=C l_{\delta}\left[C l_{\delta}(A) \mathrm{I} C l_{\delta}(X-A)\right] \subseteq$ $C l_{\delta}\left[C l_{\delta}(A)\right] \mathrm{I} C l_{\delta}\left[C l_{\delta}(X-A)\right]$

$=C l_{\delta}(A) \mathrm{I} C l_{\delta}(X-A)=F r_{\delta}(A)$. Hence $F r_{\delta}(A)$ is $\delta$-closed.

(10) $F r_{\delta}\left[F r_{\delta}(A)\right]=C l_{\delta}\left[F r_{\delta}(A)\right] \operatorname{I} C l_{\delta}\left[X-F r_{\delta}(A)\right] \subseteq$ $C l_{\delta}\left[F r_{\delta}(A)\right]=F r_{\delta}(A)$

(12) $F r_{\delta}\left[C l_{\delta}(A)\right]=C l_{\delta}\left[C l_{\delta}(A)\right]-\operatorname{Int}_{\delta}\left[C l_{\delta}(A)\right]=$ $C l_{\delta}(A)-\operatorname{Int}_{\delta}\left[C l_{\delta}(A)\right] \subseteq\left[C l_{\delta}(A)-\operatorname{Int}_{\delta}(A)\right]=F r_{\delta}(A)$.

(13) $A-F r_{\delta}(A)=A-\left[C l_{\delta}(A)-\operatorname{Int}_{\delta}(A)\right]=\operatorname{Int}_{\delta}(A)$.

Remark 1.11. Let $A$ and $B$ be subsets of $X$. Then $A \subseteq B$ does not imply that either $F r_{\delta}(B) \subseteq F r_{\delta}(A)$ or $F r_{\delta}(A) \subseteq F r_{\delta}(B)$.

Def inition 1.12. $\operatorname{Ext}_{\delta}(A)=\operatorname{Int}_{\delta}(X-A)$ is said to be a $\delta$-exterior of $A$.

Theorem 1.13. For a subset $A$ of a space $X$, the following statements hold: 
(1) $\operatorname{Ext}_{\delta}(A) \subseteq \operatorname{Ext}(A)$ where $\operatorname{Ext}(A)$ denotes the exterior of $A$;

(2) $\operatorname{Ext}_{\delta}(A)$ is $\delta-o p n$;

(3) $\operatorname{Ext}_{\delta}(A)=\operatorname{Int}_{\delta}(X-A)=X-C l_{\delta}(A)$;

(4) $\operatorname{Ext}_{\delta}\left[\operatorname{Ext}_{\delta}(A)\right]=\operatorname{Int}_{\delta}\left[C l_{\delta}(A)\right]$;

(5) If $A \subseteq B$, then $\operatorname{Ext}_{\delta}(B) \subseteq \operatorname{Ext}_{\delta}(A)$;

(6) $\operatorname{Ext}_{\delta}(A \mathrm{U} B)=\operatorname{Ext}_{\delta}(A) \mathrm{U} E x t_{\delta}(B)$;

(7) $\operatorname{Ext}_{\delta}(A) \mathrm{I} E x t_{\delta}(B) \subseteq \operatorname{Ext}_{\delta}(A \mathrm{I} B)$;

(8) $\operatorname{Ext}_{\delta}(X)=\phi$;

(9) $\operatorname{Ext}_{\delta}(\phi)=X$;

(10) $\operatorname{Ext}_{\delta}(A)=\operatorname{Ext}_{\delta}\left[X-\operatorname{Ext}_{\delta}(A)\right]$;

(11) $\operatorname{Int}_{\delta}(A) \subseteq \operatorname{Ext}_{\delta}\left[\operatorname{Ext}_{\delta}(A)\right]$;

(12) $X=\operatorname{Int}_{\delta}(A) \mathrm{U} E x t_{\delta}(A) \mathrm{U} F r_{\delta}(A)$;

(13) $\operatorname{Ext}_{\delta}(A) \mathrm{U} E x t_{\delta}(B) \subseteq \operatorname{Ext}_{\delta}(A \mathrm{I} B)$.

Proof. (4) $E x t_{\delta}\left[E x t_{\delta}(A)\right]=E x t_{\delta}\left[X-C l_{\delta}(A)\right]=$ $\operatorname{Int}_{\delta}\left[X-\left(X-C l_{\delta}(A)\right)\right]=\operatorname{Int}_{\delta}\left[C l_{\delta}(A)\right]$

(10) $\operatorname{Ext}_{\delta}\left[X-\operatorname{Ext}_{\delta}(A)\right]=\operatorname{Ext}_{\delta}\left[X-\operatorname{Int}_{\delta}(X-A)\right]$

$=\operatorname{Int}_{\delta}\left[X-\left(X-\operatorname{Int}_{\delta}(X-A)\right)\right]$

$=\operatorname{Int}_{\delta}\left[\operatorname{Int}_{\delta}(X-A)\right]=\operatorname{Int}_{\delta}(X-A)=\operatorname{Ext}_{\delta}(A)$.

(11) $\operatorname{Int}_{\delta}(A) \subseteq \operatorname{Int}_{\delta}\left[\operatorname{Cl}_{\delta}(A)\right]=\operatorname{Int}_{\delta}\left[X-\operatorname{Int}_{\delta}(X-A)\right]$ $=\operatorname{Int}_{\delta}\left[X-\operatorname{Ext}_{\delta}(A)\right]=\operatorname{Ext}_{\delta}\left[\operatorname{Ext}_{\delta}(A)\right]$.

$$
\begin{aligned}
& \operatorname{Ext}_{\delta}(A) \mathrm{U} \operatorname{Ext}_{\delta}(B)=\operatorname{Int}_{\delta}(X-A) \operatorname{UInt}_{\delta}(X-B) \\
& \subseteq \operatorname{Int}_{\delta}[(X-A) \mathrm{U}(X-B)] \\
& =\operatorname{Int}_{\delta}[X-(A \mathrm{I} B)]=\operatorname{Ext}_{\delta}(A \mathrm{I} B) .
\end{aligned}
$$

Def inition 1.14. Let $X$ be a topological space. A set $A \subseteq X$ is said to be $\delta$-saturated if for every $x \in A$ it implies that $C l_{\delta}(\{x\}) \subseteq A$. The class of all $\delta$-saturated sets in $X$ will be denoted by $B_{\delta}(X)$.

Theorem 1.15. Let $X$ be a topological space. Then $B_{\delta}(X)$ is a complete Boolean set Algebra.

Proof. We will prove that all the unions and complements of elements of $B_{\delta}(X)$ are members of $B_{\delta}(X)$. Obviously, only the proof regarding the complements is not trivial. Let $A \in B_{\delta}(X)$ and suppose that $C l_{\delta}(\{x\}) \not \subset(X-A)$ for some $x \in(X-A)$. Then there exists $y \in A$ such that $y \in C l_{\delta}(\{x\})$. It follows that $x$ and $y$ have no disjoint neighborhoods. Then $x \in C_{\delta}(\{y\})$. But this is a contradiction, because by the definition of $B_{\delta}(X)$ we have $C l_{\delta}(\{y\}) \subseteq A$. Hence, $\quad C l_{\delta}(\{x\}) \subseteq(X-A)$ for every $\quad x \in(X-A), \quad$ which implies $(X-A) \in B_{\delta}(X)$.

Corrolary 1.16. $B_{\delta}(X)$ contains every union and every intersection of $\delta$-closed and $\delta$-open sets in $X$.

Def inition 1.17. A space $X$ is said to be $\delta$-Hausdorff if for every $x \neq y \in X$, there exist $\delta$-open sets $U_{x}, V_{y}$ such that $x \in U_{x}$, $y \in V_{v}$ and $U_{x} \mathrm{I} V_{y}=\phi$.

Theorem 1.18. Let $(X, \tau)$ be a topological space. Then the following statements are equivalent:

(1) $X$ is $\delta-\mathrm{T}_{2}$;

(2) Let $x \in X$. For each $y \neq x$, there exists a $\delta$-open set $U$ such that $x \in U$ and $y \notin C l_{\delta}(U)$; 


$$
\text { For each } \quad x \in X \text {, }
$$

I $\left\{C l_{\delta}(U) \mid U \in \tau_{\delta}\right.$ and $\left.x \in U\right\}=\{x\}$;

(4) The diagonal $\Delta=\{(x, x) \mid x \in X\}$ is a $\delta$-closed set in $X \times X$.

Proof. $(1) \Rightarrow(2)$ : Let $x \in X$ and $y \neq x$. Then there are disjoint $\delta$-open sets $U$ and $V$ such that $x \in U$ and $y \in V$. Clearly, $V^{c}$ is $\delta$-closed, $C l_{\delta}(U) \subseteq V^{c}, \quad y \notin V^{c}$ and therefore $y \notin C l_{\delta}(U)$.

$(2) \Rightarrow(3)$. If $y \neq x$, there exists a $\delta$-open set $U$ such that $x \in U$ and $y \notin C l_{\delta}(U)$. So $y \notin \mathrm{I}\left\{C l_{\delta}(U) \mid U \in \tau_{\delta}\right.$ and $\left.x \in U\right\}$.

$(3) \Rightarrow(4)$. We prove that $\Delta^{c}$ is $\delta$-open. Let $(x, y) \notin \Delta$. Then $y \neq x$ and since I $\left\{C l_{\delta}(U) \mid U \in \tau_{\delta}\right.$ and $\left.x \in U\right\}=\{x\}$ there is some $U \in \tau_{\delta} \quad$ with $\quad x \in U$ and $\quad y \notin C l_{\delta}(U)$. Since $U \mathrm{I}\left[C l_{\delta}(U)\right]^{c}=\phi, \quad U \times\left[C l_{\delta}(U)\right]^{c}$ is a $\delta$-open set such that $(x, y) \in U \times\left[C l_{\delta}(U)\right]^{c} \subseteq \Delta^{c}$.

$(4) \Rightarrow(1)$. If $y \neq x$, then $(x, y) \notin \Delta$ and thus there exist $\delta$-open sets $U$ and $V$ such that $(x, y) \in U \times V$ and $(U \times V) \mathrm{I} \Delta=\phi$. Clearly, for the $\delta$-open sets $U$ and $V$ we have: $x \in U, y \in V$ and $U$ I $V=\phi$.

Def inition 1.19. A subset $A$ of a space $X$ is said to be $\delta$-compact if every cover of $X$ by $\delta$-open sets has a finite subcover.

It is well-known that every closed subset of a compact space is compact. The next theorem approximates this result for $\delta$-compactness.

Theorem 1.20. A $\delta$-compact subset of a $\delta$-Hausdorff space is $\delta$-closed.

Proof. Let A be a $\delta$-compact subset of a $\delta$-Hausdorff space $X$.We will show that
$(X-A)$ is $\delta$-open. Let $x \in(X-A)$. Then for each $a \in A$ there exist $\delta$-open sets $U_{x, a}$ and $V_{a}$ such that $x \in U_{x, a}$ and $a \in V_{a}$ and $U_{x, a}$ I $V_{a}=\phi$. The collection $\left\{V_{a} \mid a \in A\right\}$ is a $\delta$-open cover of $A$. Therefore, there exists a finite subcollection $\left\{V_{a_{k}} \mid k=1,2,3, \ldots, n\right\}$ that covers $A$. Let $U_{x}=\mathrm{I}\left\{U_{x, a_{k}} \mid k=1,2,3, \ldots, n\right\}$. Then $x \in U_{x}, U_{x}$ is $\delta$ open and $U_{x} \mathrm{I} A=\phi$. This proves that $A$ is $\delta$-closed .

Theorem 1.21. A $\delta$-closed subset of a $\delta$-Hausdorff space is $\delta$-compact.

Proof. Let $X$ be $\delta$-compact and let $A$ be a $\delta$-closed subset of $X$. Let $\Gamma$ be a $\delta$-open cover of $A$. Then $\Gamma^{*}=\Gamma \mathrm{U}\{X-A\}$ is a $\delta$-open cover of $X$. Since $X$ is $\delta$-compact, this collection $\Gamma^{*}$ has a finite collection $\Lambda^{*}$ that covers $X$. But then $\Gamma$ ha a finite subcollection $\Lambda=\Lambda^{*}-\{X-A\}$ that covers $A$ as we need.

Def inition 1.22. Let $A$ be a subset of a topological space $X$. Then $\delta$-kernel of $A$, denoted by $\operatorname{Ker}_{\delta}(A)=\mathrm{I}\left\{O \in \tau_{\delta} \mid A \subseteq O\right\}$.

Def inition 1.23. Let $x$ be a point of a topological space $X$. Then $\delta$-kernel of $x$, denoted by $\operatorname{Ker}_{\delta}(\{x\})$ is defined to be the set $\operatorname{Ker}_{\delta}(\{x\})=\mathrm{I}\left\{O \in \tau_{\delta} \mid x \in O\right\}$.

Lemma 1.24. Let $(X, \tau)$ be a topological space and $x \in X$.

Then $\operatorname{Ker}_{\delta}(A)=\left\{x \in X \mid C l_{\delta}(\{x\}) \mathrm{I} A \neq \phi\right\}$.

Proof. Let $x \in \operatorname{Ker}_{\delta}(A)$ and $\quad C_{\delta}(\{x\}) \mathrm{I} A=\phi$. Hence $x \notin\left[X-\operatorname{Ker}_{\delta}(\{x\})\right]$ which is a $\delta$-open set containing $A$. This is impossible, since $x \in \operatorname{Ker}_{\delta}(A)$. Consequently, $C l_{\delta}(\{x\})$ I $A \neq \phi$. Let $C l_{\delta}(\{x\}) \mathrm{I} A \neq \phi \quad$ and $x \notin \operatorname{Ker}_{\delta}(A)$. Then there exists a $\delta$-open set $D$ containing $A$ and 
$x \notin D$. Let $y \in C l_{\delta}(\{x\}) \mathrm{I} A$. Hence, $D$ is a $\delta$-open neighborhood of $y$ with $x \notin D$. By this contradiction, $x \in \operatorname{Ker}_{\delta}(A)$ and the claim.

Def inition 1.25. A topological space $(X, \tau)$ is said to be a $\delta-R_{0}$ space if every $\delta$-open set contains the $\delta$-closure of each of its singletons.

Lemma 1.26. Let $(X, \tau)$ be a topological space and $x \in X$. Then $y \in \operatorname{Ker}_{\delta}(\{x\})$ if and only if $x \in \operatorname{Ker}_{\delta}(\{y\})$.

Proof. Suppose that $y \notin \operatorname{Ker}_{\delta}(\{x\})$. Then there exists a $\delta$-open set $V$ containing $x$ such that $y \notin V$. Therefore we have $x \notin C l_{\delta}(\{y\})$. The proof of the converse case can be done similarly.

Lemma 1.27. The following statements are equivalent for any points $x$ and $y$ in a topological space $(X, \tau)$ :

(1) $\operatorname{Ker}_{\delta}(\{x\}) \neq \operatorname{Ker}_{\delta}(\{y\})$;

(2) $C l_{\delta}(\{x\}) \neq C l_{\delta}(\{y\})$.

Proof. $\quad(1) \Rightarrow(2): \quad$ Suppose that $\operatorname{Ker}_{\delta}(\{x\}) \neq \operatorname{Ker}_{\delta}(\{y\})$. Then there exists a point $z$ in $X$ such that $z \in \operatorname{Ker}_{\delta}(\{x\})$ and $z \notin \operatorname{Ker}_{\delta}(\{y\})$. It follows from $z \in \operatorname{Ker}_{\delta}(\{x\})$ that $\quad\{x\} \mathrm{I} C l_{\delta}(\{x\}) \neq \phi$. This implies that $x \in C_{\delta}(\{z\})$. By $z \notin \operatorname{Ker}_{\delta}(\{y\})$, we have

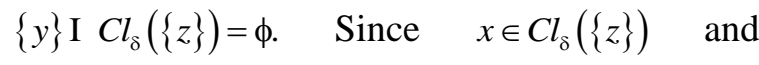
$C l_{\delta}(\{x\}) \subseteq C l_{\delta}(\{z\})$. Hence $\quad\{y\} \mathrm{I} C l_{\delta}(\{x\})=\phi$. Therefore, $C l_{\delta}(\{x\}) \neq C l_{\delta}(\{y\})$.

$(2) \Rightarrow(1):$ Suppose that $\quad C_{\delta}(\{x\}) \neq C l_{\delta}(\{y\})$. Then there exists a point $z \in X$ such that $z \in C l_{\delta}(\{x\})$ and $z \notin C l_{\delta}(\{y\})$. Then, there exists a $\delta$-open set containing $z$ and therefore $x$ but not $y, \quad$ i.e., $\quad y \notin \operatorname{Ker}_{\delta}(\{x\})$. Hence $\operatorname{Ker}_{\delta}(\{x\}) \neq \operatorname{Ker}_{\delta}(\{y\})$.

Theorem 1.28. A topological space $(X, \tau)$ is a $\delta-R_{0}$ space if and only if for every $x$ and $y$ in $X . \quad C l_{\delta}(\{x\}) \neq C l_{\delta}(\{y\})$ implies $C l_{\delta}(\{x\}) \mathrm{I} C l_{\delta}(\{y\})=\phi$.

Proof. Necessity. Suppose that $(X, \tau)$ is $\delta-R_{0}$ and $x, y \in X$ such that $C l_{\delta}(\{x\}) \neq C l_{\delta}(\{y\})$. Then there exists $z \in C l_{\delta}(\{x\})$ such that $z \notin C l_{\delta}(\{y\})$ (or $z \in C l_{\delta}(\{y\})$ such that $z \notin C l_{\delta}(\{x\})$. There exists $V \in \tau_{\delta}$ such that $y \notin V$ and $z \in V$; hence $x \in V$. Therefore, we have $x \notin C l_{\delta}(\{y\})$. Thus $x \in\left[X-C l_{\delta}(\{y\})\right] \in \tau_{\delta}, \quad$ which implies $C l_{\delta}(\{x\}) \subseteq\left[X-C l_{\delta}(\{y\})\right] \quad$ and $C l_{\delta}(\{x\}) \mathrm{I} C l_{\delta}(\{y\})=\phi$. The proof for otherwise is similar.

Sufficiency. Let $V \in \tau_{\delta}$ and let $x \in V$. We will show that $C l_{\delta}(\{x\}) \subseteq V$. Let $y \notin V$, i.e., $y \in(X-V)$. Then $x \neq y$ and $x \notin C l_{\delta}(\{y\})$. This shows that $C l_{\delta}(\{x\}) \neq C l_{\delta}(\{y\})$. By assumption, $C l_{\delta}(\{x\}) \mathrm{I} C l_{\delta}(\{y\})=\phi$. Hence $y \notin C l_{\delta}(\{x\})$ and therefore $C l_{\delta}(\{x\}) \subseteq V$.

Theorem 1.29. A topological space $(X, \tau)$ is a $\delta-R_{0}$ space if and only if for any points $x$ and $y \quad$ in $X . \quad \operatorname{Ker}_{\delta}(\{x\}) \neq \operatorname{Ker}_{\delta}(\{y\}) \quad$ implies $\operatorname{Ker}_{\delta}(\{x\}) \neq \operatorname{Ker}_{\delta}(\{y\})$.

Proof. Suppose that $(X, \tau)$ is a $\delta-R_{0}$ space. Thus by Lemma 1.27, for any points $x$ and $y$ in $X$ if $\operatorname{Ker}_{\delta}(\{x\}) \neq \operatorname{Ker}_{\delta}(\{y\})$ then $C l_{\delta}(\{x\}) \neq C l_{\delta}(\{y\})$. Now we prove that $\operatorname{Ker}_{\delta}(\{x\}) \mathrm{I} \operatorname{Ker}_{\delta}(\{y\})=\phi$. Assume that $z \in \operatorname{Ker}_{\delta}(\{x\}) \mathrm{I} \operatorname{Ker}_{\delta}(\{y\})$. By $z \in \operatorname{Ker}_{\delta}(\{x\})$ and 
Lemma 1.26, it follows that $x \in \operatorname{Ker}_{\delta}(\{z\})$. Since $x \in \operatorname{Ker}_{\delta}(\{x\}), \quad$ by $\quad$ Theorem 1.28, $C l_{\delta}(\{x\})=C l_{\delta}(\{z\})$. Similarly, we have $C l_{\delta}(\{y\})=C l_{\delta}(\{z\})=C l_{\delta}(\{x\})$. This $\quad$ is $\quad$ a contradiction. Therefore, we have $\operatorname{Ker}_{\delta}(\{x\}) \mathrm{I} \operatorname{Ker}_{\delta}(\{y\})=\phi$.

Conversely, let $(X, \tau)$ be a topological space such that for any points $x$ and $y$ in $X$, $\operatorname{Ker}_{\delta}(\{x\}) \neq \operatorname{Ker}_{\delta}(\{y\}) \quad$ implies $\operatorname{Ker}_{\delta}(\{x\}) \mathrm{I} \operatorname{Ker}_{\delta}(\{y\})=\phi$. If $C l_{\delta}(\{x\}) \neq C l_{\delta}(\{y\})$, then by Lemma 1.27, $\operatorname{Ker}_{\delta}(\{x\}) \neq \operatorname{Ker}_{\delta}(\{y\})$. Hence $\operatorname{Ker}_{\delta}(\{x\}) \operatorname{I} \operatorname{Ker}_{\delta}(\{y\})=\phi$ which implies $C l_{\delta}(\{x\}) \mathrm{I} C l_{\delta}(\{y\})=\phi . \quad$ Because $\quad z \in \operatorname{Ker}_{\delta}(\{x\})$ implies that $x \in \operatorname{Ker}_{\delta}(\{z\})$. Therefore $\operatorname{Ker}_{\delta}(\{x\}) \mathrm{I} \operatorname{Ker}_{\delta}(\{y\}) \neq \phi$. By hypothesis, we have $\operatorname{Ker}_{\delta}(\{x\})=\operatorname{Ker}_{\delta}(\{z\})$ Then $z \in C l_{\delta}(\{x\}) \mathrm{I} C l_{\delta}(\{y\}) \quad$ implies that $\operatorname{Ker}_{\delta}(\{x\})=\operatorname{Ker}_{\delta}(\{z\})=\operatorname{Ker}_{\delta}(\{y\})$. This is a

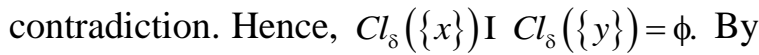
Theorem $1.28(X, \tau)$ is a $\delta-R_{0}$ space.

Theorem 1.30. For a topological space $(X, \tau)$, the following properties are equivalent:

(1) $(X, \tau)$ is a $\delta-R_{0}$ space;

(2) For any $A \neq \phi$ and $G \in \tau_{\delta}$ such that $A$ I $G \neq \phi$, there exists $F \in C_{\delta}(X, \tau)$ such that $A$ I $F \neq \phi$ and $F \subseteq G$;

(3) Any $G \in \tau_{\delta}, G=\mathrm{U}\left\{F \in C_{\delta}(X, \tau) \mid F \subseteq G\right\}$;

(4)Any $F \in C_{\delta}\left(X, \tau_{\delta}\right), F=\mathrm{I}\left\{G \in \tau_{\delta} \mid F \subseteq G\right\}$;

(5) For any $x \in X, C l_{\delta}(\{x\}) \subseteq \operatorname{Ker}_{\delta}(\{x\})$.

Proof. $(1) \Rightarrow(2)$ : Let $A$ be a nonempty subset of $X$ and $G \in \tau_{\delta}$ such that $A$ I $G \neq \phi$. There exists $x \in A$ I $G$. Since $x \in G \in \tau_{\delta}, C l_{\delta}(\{x\}) \subseteq G$. Set $F=C l_{\delta}(\{x\})$. Then $F$ is a $\delta$-closed subset of $X$ such that $F \subseteq G$ and $A$ I $F \neq \phi$.

$(2) \Rightarrow(3): \quad$ Let $\quad G \in \tau_{\delta}$. Then $\mathrm{U}\left\{F \in C_{\delta}(X, \tau) \mid F \subseteq G\right\} \subseteq G$. Let $x$ be any point of $G$. There exists $F \in C_{\delta}(X, \tau)$ such that $x \in F$ and $F \subseteq G$. Therefore, we have $x \in F \subseteq \mathrm{U}\left\{F \in C_{\delta}(X, \tau) \mid F \subseteq G\right\} \quad$ and hence $G=\mathrm{U}\left\{F \in C_{\delta}(X, \tau) \mid F \subseteq G\right\}$.

$(3) \Rightarrow(4)$ : This is obvious.

$(4) \Rightarrow(5)$ : Let $x$ be any point of $X$ and $y \notin \operatorname{Ker}_{\delta}(\{x\})$. There exists $V \in \tau_{\delta}$ such that $x \in V$ and $y \notin V$; hence $C l_{\delta}(\{x\})$ I $V=\phi$. By $(4)$ (I $\left.\left\{G \in \tau_{\delta} \mid C l_{\delta}(\{y\}) \subseteq G\right\}\right) \mathrm{I} V=\phi$. There exists $G \in \tau_{\delta} \quad$ such that $x \notin G$ and $C l_{\delta}(\{y\}) \subseteq G$. Therefore $\quad C l_{\delta}(\{x\}) \mathrm{I} G=\phi \quad$ and $y \notin C l_{\delta}(\{x\})$. Consequently, we obtain $C_{\delta}(\{x\}) \subseteq \operatorname{Ker}_{\delta}(\{x\})$.

$(5) \Rightarrow(1): \quad$ Let $\quad G \in \tau_{\delta} \quad$ and $\quad x \in G$. Suppose $y \in \operatorname{Ker}_{\delta}(\{x\})$. Then $x \in C l_{\delta}(\{y\})$ and $y \in G$. This implies that $C l_{\delta}(\{x\}) \subseteq \operatorname{Ker}_{\delta}(\{x\}) \subseteq G$. Therefore, $(X, \tau)$ is a $\delta-R_{0}$ space.

Corollary 1.31. For a topological space $(X, \tau)$, the following properties are equivalent:

(1) $(X, \tau)$ is a $\delta-R_{0}$ space;

(2) $\operatorname{Cl}_{\delta}(\{x\})=\operatorname{Ker}_{\delta}(\{x\})$ for all $x \in X$.

Proof. $(1) \Rightarrow(2)$ : Suppose that $(X, \tau)$ is a $\delta-R_{0} \quad$ space. $\quad$ By $\quad$ Theorem 1.30 , $C l_{\delta}(\{x\}) \subseteq \operatorname{Ker}_{\delta}(\{x\})$ for each $x \in X$. Let $y \in \operatorname{Ker}_{\delta}(\{x\})$. Then $x \in C_{\delta}(\{y\})$ and so $C l_{\delta}(\{x\})=C l_{\delta}(\{y\})$. Therefore, $y \in C l_{\delta}(\{x\})$ and 
hence $\operatorname{Ker}_{\delta}(\{x\}) \subseteq C l_{\delta}(\{x\})$. This shows that $\mathrm{Cl}_{\delta}(\{x\})=\operatorname{Ker}_{\delta}(\{x\})$.

$(2) \Rightarrow(1)$ : This is obvious by Theorem 1.30.

Theorem 1.32. For a topological space $(X, \tau)$, the following properties are equivalent:

(1) $(X, \tau)$ is a $\delta-R_{0}$ space;

(2) $x \in C_{\delta}(\{y\})$ if and only if $y \in C l_{\delta}(\{x\})$, for any points $x$ and $y$ in $X$.

Proof. $(1) \Rightarrow(2)$ : Assume that $X$ is $\delta-R_{0}$. Let $x \in C l_{\delta}(\{y\})$ and $D$ be any $\delta$-open set such that $y \in D$. Now by hypothesis, $x \in D$. Therefore, every $\delta$-open set containing $y$ contains $x$. Hence $y \in C l_{\delta}(\{x\})$.

$(2) \Rightarrow(1)$ : Let $U$ be a $\delta$-open set and $x \in U$. If $y \notin U$, then $x \notin C l_{\delta}(\{y\})$ and hence $y \notin C l_{\delta}(\{x\})$. This implies that $C l_{\delta}(\{x\}) \subseteq U$. Hence $(X, \tau)$ is $\delta-R_{0}$.

Theorem 1.33. For a topological space $(X, \tau)$, the following properties are equivalent:

(1) $(X, \tau)$ is a $\delta-R_{0}$ space;

(2)

If $F$ is $\delta$-closed, then $F=\operatorname{Ker}_{\delta}(F)$.

(3) If $F$ is $\delta$-closed and $x \in F$, then $\operatorname{Ker}_{\delta}(F) \subseteq F$.

(4)If $x \in X$, then $\operatorname{Ker}_{\delta}(\{x\}) \subseteq C l_{\delta}(\{x\})$.

Proof. (1) $\Rightarrow(2)$ : Let $F$ be a $\delta$-closed set and $x \notin F$. Thus $(X-F)$ is a $\delta$-open set containing $x$. Since $(X, \tau)$ is $\delta-R_{0} . \quad C l_{\delta}(\{x\}) \subseteq(X-F)$.
Thus $\quad l_{\delta}(\{x\}) \mathrm{I} F=\phi$ and by Lemma 1.24 $x \notin \operatorname{Ker}_{\delta}(F)$. Therefore $\operatorname{Ker}_{\delta}(F)=F$.

$(2) \Rightarrow(3): \quad$ In general, $\quad A \subseteq B \quad$ implies $\operatorname{Ker}_{\delta}(A) \subseteq C l_{\delta}(B)$. Therefore, it follows from (2) that $\operatorname{Ker}_{\delta}(\{x\}) \subseteq \operatorname{Ker}_{\delta}(F)=F$.

(3) $\Rightarrow(4)$ : Since $x \in C l_{\delta}(\{x\})$ and $C l_{\delta}(\{x\})$ is $\delta$-closed, by (3), $\operatorname{Ker}_{\delta}(\{x\}) \subseteq C l_{\delta}(\{x\})$.

$(4) \Rightarrow(1)$ We show the implication by using Theorem 3.19. Let $x \in C l_{\delta}(\{y\})$. Then by Lemma 1.26, $y \in \operatorname{Ker}_{\delta}(\{x\})$. Since $x \in C_{\delta}(\{x\})$ and $C l_{\delta}(\{x\})$ is a $\delta$-closed set, by (4) we obtain $y \in \operatorname{Ker}_{\delta}(\{x\}) \subseteq C l_{\delta}(\{x\})$. Therefore $x \in C l_{\delta}(\{y\})$ implies $y \in C l_{\delta}(\{x\})$. The converse is obvious and $(X, \tau)$ is $\delta-R_{0}$.

Theorem 1.34. Let $(X, \tau)$ be a topological space. Then I $\left\{C l_{\delta}(\{x\}) \mid x \in X\right\}=\phi$ if and only if $\operatorname{Ker}_{\delta}(\{x\}) \neq X$ for every $x \in X$.

Proof. Necessity. Suppose that I $\left\{C l_{\delta}(\{x\}) \mid x \in X\right\}=\phi$. Assume that there is a point $y$ in $X$ such that $\operatorname{Ker}_{\delta}(\{y\})=X$. Then $y \notin O$, where $O$ is some proper $\delta$-open subset of $X$. This implies that $y \in \mathrm{I}\left\{C l_{\delta}(\{x\}) \mid x \in X\right\}$. But this is a contradiction.

Sufficiency. Assume that $\operatorname{Ker}_{\delta}(\{x\}) \neq X$ for every $x \in X$. If there exists a point $y \in X$ such that $y \in \mathrm{I}\left\{C l_{\delta}(\{x\}) \mid x \in X\right\}$, then every $\delta$-open set containing $y$ must contain every point of $X$. This implies that the space $X$ is the unique $\delta$-open set containing $y$. Hence 
$\operatorname{Ker}_{\delta}(\{x\})=X$ which is a contradiction.

Therefore, I $\left\{C l_{\delta}(\{x\}) \mid x \in X\right\}=\phi$.

Def inition 1.35. A filter base $F$ is called $\delta$-convergent to a point $x$ in $X$, if for any $\delta$-open set $U$ of $X$ containing $X$, there exists $B$ in $F$ such that $B$ is a subset of $U$.

Lemma 1.36. Let $(X, \tau)$ be a topological space and $x$ and $y$ be any two points in $X$ such that every net in $X \delta$-converging to $y$ $\delta$-converges to $x$. Then $x \in C_{\delta}(\{y\})$.

Proof. Suppose that $x_{\alpha}=y$ for $\alpha \in I$. Then $\left\{x_{\alpha}: \alpha \in I\right\}$ is a net in $C l_{\delta}(\{y\})$. Since $\left\{x_{\alpha}: \alpha \in I\right\} \delta$-converges to $y$, so $\left\{x_{\alpha}: \alpha \in I\right\}$ $\delta$-converges to $x$ and this implies that $x \in C l_{\delta}(\{y\})$.

Theorem 1.37. For a topological space $(X, \tau)$, the following statements are equivalent:

(1) $(X, \tau)$ is $\delta-R_{0}$ space;

(2) If $x, y \in X$, then $y \in C l_{\delta}(\{x\})$ if and only if every net in $\mathrm{X} \delta$-converging to $y$ $\delta$-converges to $x$.

Proof. $(1) \Rightarrow(2)$ : Let $x, y \in X$ such that $y \in C l_{\delta}(\{x\})$. Suppose that $\left\{x_{\alpha}: \alpha \in I\right\}$ is a net in $X$ such that this net $\delta$-converges to $y$. Since $y \in C l_{\delta}(\{x\})$ so by Theorem 1.28 we have $\quad C l_{\delta}(\{x\})=C l_{\delta}(\{y\})$. Therefore $x \in C l_{\delta}(\{y\})$. This means that the net $\left\{x_{\alpha}: \alpha \in I\right\} \delta$-converges to $x$.

Conversely, let $x, y \in X$ such that every net in $X \delta$-converging to $y \delta$-converges to $x$. Then $x \in C l_{\delta}(\{y\})$ by Lemma 1.36. By
Theorem 1.28, we have $C l_{\delta}(\{x\})=C l_{\delta}(\{y\})$. Therefore $y \in C l_{\delta}(\{x\})$.

$(2) \Rightarrow(1)$ : Assume that $x$ and $y$ are any two points of $X$ such that

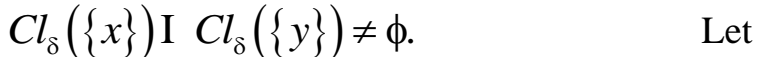
$z \in C l_{\delta}(\{x\}) \mathrm{I} C l_{\delta}(\{y\})$. So there exists a net $\left\{x_{\alpha}: \alpha \in I\right\} \quad$ in $C l_{\delta}(\{x\})$ such that $\left\{x_{\alpha}: \alpha \in I\right\} \delta$-converges to $z$. Since $z \in C l_{\delta}(\{y\})$. So by hypothesis $\left\{x_{\alpha}: \alpha \in I\right\}$ $\delta$-converges to $y$. It follows that $y \in C l_{\delta}(\{x\})$. Similarly we obtain $x \in C l_{\delta}(\{y\}) . \quad$ Therefore $C l_{\delta}(\{x\})=C l_{\delta}(\{y\})$ and by Theorem 1.28, $(X, \tau)$ is $\delta-R_{0}$.

\section{CHARACTERIZATIONS OF MAPPINGS}

The purpose of this part is to explore properties and characterizations of $\delta$-continuous, $\delta$-irresolute, $\delta$-open, $\delta$-closed, pre $-\delta-$ open, and pre $-\delta-$ closed functions.

\subsection{DELTA - CONTINUOUS FUNCTIONS}

The purpose of this section is to investigate properties and characterizations of $\delta$-continuous functions.

Definition 2.1. A function $f:(X, \tau) \rightarrow(Y, \sigma)$ is said to be $\delta$-continuous if $f^{-1}(V) \in \tau_{\delta}$ for every $V \in \sigma$.

Theorem 2.2. Let $\quad f:(X, \tau) \rightarrow(Y, \sigma)$ be a function. Then the following are equivalent:

(1) $f$ is $\delta$-continuous;

(2) The inverse image of each closed set in $\mathrm{Y}$ is a $\delta$-closed set in $\mathrm{X}$; 
(3) $\quad C l_{\delta}\left[f^{-1}(V)\right] \subseteq f^{-1}[C l(V)]$, for every $V \subseteq Y$;

(4) $f\left[C l_{\delta}(U)\right] \subseteq C l[f(U)]$, for every $U \subseteq X$;

(5) For any point $x \in X$ and any open set $V$ of $Y$ containing $f(x)$, there exists $U \in \tau_{\delta}$ such that $x \in U$ and $f(U) \subseteq V$;

(6) $B d_{\delta}\left[f^{-1}(V)\right] \subseteq f^{-1}[B d(V)]$, for every $V \subseteq Y$;

(7) $f\left[D_{\delta}(U)\right] \subseteq C l[f(U)]$, for every $U \subseteq X$;

(8) $f^{-1}[\operatorname{Int}(V)] \subseteq \operatorname{Int}_{\delta}\left[f^{-1}(V)\right], \quad$ for every $V \subseteq Y$;

Proof. (1) $\Rightarrow(2)$ : Let $F \subseteq Y$ be closed. Since $f$ is $\delta$-continuous, $f^{-1}(Y-F)=X-f^{-1}(F)$ is $\delta$-open. Therefore, $f^{-1}(F)$ is $\delta$-closed in $X$.

$(2) \Rightarrow(3)$ : Since $C l(V)$ is closed for every $V \subseteq Y$, then $\quad f^{-1}[C l(V)] \quad$ is $\delta$-closed. Therefore $f^{-1}[C l(V)]=C l_{\delta}\left[f^{-1}(C l(V))\right] \supseteq C l_{\delta}\left[f^{-1}(V)\right]$.

(3) $\Rightarrow(4)$ : Let $U \subseteq X$ and $f(U)=V$. Then $C l_{\delta}\left[f^{-1}(V)\right] \subseteq f^{-1}[C l(V)] . \quad$ Thus $C l_{\delta}(U) \subseteq C l_{\delta}\left[f^{-1}(f(U))\right] \subseteq f^{-1}[C l(f(U))]$ and $f\left[C l_{\delta}(U)\right] \subseteq C l[f(U)]$.

(4) $\Rightarrow(2)$ : Let $W \subseteq Y$ be a closed set, and $U=f^{-1}(W) . \quad$ Then $\quad f\left[C l_{\delta}(U)\right] \subseteq C l[f(U)]$ $=C l\left[f\left(f^{-1}(W)\right)\right] \subseteq C l(W)=W$. Thus

$C l_{\delta}(U) \subseteq f^{-1}\left[f\left(C l_{\delta}(U)\right)\right] \subseteq f^{-1}(W)=U$. So $U$ is $\delta$-closed.

(2) $\Rightarrow(1):$ Let $V \subseteq Y$ be an open set. Then $Y-V$ is closed. Then $f^{-1}(Y-V)=X-f^{-1}(V)$ is $\delta$-closed in $X$ and hence $f^{-1}(V)$ is $\delta$-open in $X$.

$(1) \Rightarrow(5): \quad$ Let $\quad f:(X, \tau) \rightarrow(Y, \sigma) \quad$ be $\delta$-continuous. For any $x \in X$ and any open set $V$ of $Y$ containing $f(x), U=f^{-1}(V) \in \tau_{\delta}$, and $f(U)=f\left[f^{-1}(V)\right] \subseteq V$.

$(5) \Rightarrow(1)$ : Let $V \in \sigma$. We prove $f^{-1}(V) \in \tau_{\delta}$. Let $x \in f^{-1}(V)$. Then $f(x) \in V$ and there exists $\quad U \in \tau_{\delta} \quad$ such that $\quad x \in U$ and $f(x) \in f(U) \subseteq V$. Hence $x \in U \subseteq f^{-1}[f(U)] \subseteq f^{-1}(V)$. It shows that $f^{-1}(V)$ is a $\delta$-neighborhood of each of its points. Therefore $f^{-1}(V) \in \tau_{\delta}$.

$(6) \Rightarrow(8)$ : Let $V \subseteq Y$. Then by hypothesis, $B d_{\delta}\left[f^{-1}(V)\right] \subseteq f^{-1}[B d(V)]$

$\Rightarrow f^{-1}(V)-\operatorname{Int}_{\delta}\left[f^{-1}(V)\right] \subseteq f^{-1}[V-\operatorname{Int}(V)]$

$=f^{-1}(V)-f^{-1}[\operatorname{Int}(V)]$

$\Rightarrow f^{-1}[\operatorname{Int}(V)] \subseteq \operatorname{Int}_{\delta}\left[f^{-1}(V)\right]$

$(8) \Rightarrow(6)$ : Let $V \subseteq Y$. Then by hypothesis, $f^{-1}[\operatorname{Int}(V)] \subseteq \operatorname{Int}_{\delta}\left[f^{-1}(V)\right]$

$\Rightarrow$

$f^{-1}(V)-\operatorname{Int}_{\delta}\left[f^{-1}(V)\right] \subseteq f^{-1}(V)-f^{-1}[\operatorname{Int}(V)]=f^{-1}[V-\operatorname{Int}(V)]$

$\Rightarrow B d_{\delta}\left[f^{-1}(V)\right] \subseteq f^{-1}[B d(V)]$.

$(1) \Rightarrow(7): \quad$ It is obvious, since $f$ is $\delta$-continuous and by $f\left[C l_{\delta}(U)\right] \subseteq C l[f(U)]$ for each $U \subseteq X$. So $f\left[D_{\delta}(U)\right] \subseteq C l[f(U)]$.

(7) $\Rightarrow(1)$ : Let $U \subseteq Y$ be an open set, $V=Y-U$ and $f^{-1}(V)=W$. Then by hypothesis $f\left[D_{\delta}(W)\right] \subseteq C l[f(W)]$.

Thus 
$f\left[D_{\delta}\left(f^{-1}(V)\right)\right] \subseteq C l\left[f\left(f^{-1}(V)\right)\right] \subseteq C l(V)=V$.

Then $D_{\delta}\left[f^{-1}(V)\right] \subseteq f^{-1}(V) \quad$ and

$f^{-1}(V)$ is $\delta$-closed.

Therefore,

$f$ is $\delta$-continuous.

$(1) \Rightarrow(8)$ : Let $\quad V \subseteq Y$. Then $\quad f^{-1}[\operatorname{Int}(V)]$ is $\delta$-open in $X$. Thus $\quad f^{-1}[\operatorname{Int}(V)]=$ $\operatorname{Int}_{\delta}\left[f^{-1}(\operatorname{Int}(V))\right] \subseteq \quad \operatorname{Int}_{\delta}\left[f^{-1}(V)\right]$. Therefore $f^{-1}[\operatorname{Int}(V)] \subseteq \operatorname{Int}_{\delta}\left[f^{-1}(V)\right]$.

$(8) \Rightarrow(1)$ : Let $V \subseteq Y$ be an open set. Then $f^{-1}(V)=f^{-1}[\operatorname{Int}(V)] \subseteq \operatorname{Int}_{\delta}\left[f^{-1}(V)\right]$. Therefore, $f^{-1}(V)$ is $\delta$-open. Hence $f$ is $\delta$-continuous.

In the next Theorem, $\# \delta-c$. denotes the set of points $x$ of $X$ for which a function $f:(X, \tau) \rightarrow(Y, \sigma)$ is not $\delta$-continuous.

Theorem 2.3. $\# \delta-c$. is identical with the union of the $\delta$-frontiers of the inverse images of $\delta$-open sets containing $f(x)$.

Proof. Suppose that $f$ is not $\delta$-continuous at a point $x$ of $X$. Then there exists an open set $V \subseteq Y$ containing $f(x)$ such that $f(U)$ is not a subset of $V$ for every $U \in \tau_{\delta}$ containing $x$. Hence, we have $U$ I $f^{-1}\left(X-f^{-1}(V)\right) \neq \phi$ for $\quad$ every $\quad U \in \tau_{\delta}$ containing $x$. It follows that $x \in C l_{\delta}\left[X-f^{-1}(V)\right]$. We also have $x \in f^{-1}(V) \subseteq C l_{\delta}\left[f^{-1}(V)\right]$. This means that $x \in F r_{\delta}\left[f^{-1}(V)\right]$. Now, let $f$ be $\delta$-continuous at $x \in X$ and $V \subseteq Y$ any open set containing $f(x)$. Then, $x \in f^{-1}(V)$ is a $\delta$-open set of $X$. Thus. $x \in \operatorname{Int}_{\delta}\left[f^{-1}(V)\right]$ and therefore $x \notin F r_{\delta}\left[f^{-1}(V)\right]$ for every open set $V$ containing $f(x)$.
Remarks 2.4. (1) Every $\delta$-continuous function is continuous but the converse may not be true.

(2)If a function $f:(X, \tau) \rightarrow(Y, \sigma)$ is $\delta$-continuous and a function $g:(Y, \sigma) \rightarrow(Z, \vartheta)$ is $\delta$-continuous, then $g \circ f:(X, \tau) \rightarrow(Z, \vartheta)$ is $\delta$-continuous.

(3) If a function $f:(X, \tau) \rightarrow(Y, \sigma)$ is $\delta$-continuous and a function $g:(Y, \sigma) \rightarrow(Z, \vartheta)$ is continuous, then $g \circ f:(X, \tau) \rightarrow(Z, \vartheta)$ is $\delta$-continuous.

(4)Let $(X, \tau)$ and $(Y, \sigma)$ be topological spaces. If $f:(X, \tau) \rightarrow(Y, \sigma)$ is a function, and one of the following

(a) $f^{-1}[\operatorname{Int}(B)] \subseteq \operatorname{Int}_{\delta}\left[f^{-1}(B)\right]$ for each $B \subseteq Y$.

(b) $C l_{\delta}\left[f^{-1}(B)\right] \subseteq f^{-1}[C l(B)]$ for each $B \subseteq Y$.

(c) $f\left[C l_{\delta}(A)\right] \subseteq C l[f(A)]$ for each $A \subseteq X$ holds, then $f$ is continuous.

Lemma 2.5. Let $A \subseteq Y \subseteq X, Y$ is $\delta$-open in $X$ and $A$ is $\delta$-open in $Y$. Then $A$ is $\delta$-open in $X$.

Proof. Since $A$ is $\delta$-open in $Y$, there exists a $\delta$-open set $U \subseteq X$ such that $A=Y$ I $U$. Thus $A$ being the intersection of two $\delta$-open sets in $X$, is $\delta$-open in $X$.

Theorem 2.6. Let $f:(X, \tau) \rightarrow(Y, \sigma)$ be a mapping and $\left\{U_{i}: i \in I\right\}$ be a cover of $X$ such that $U_{i} \in \tau_{\delta}$ for each $i \in I$. Then prove that $f$ is $\delta$-continuous.

Proof. Let $V \subseteq Y$ be an open set, then $\left(f \mid U_{i}\right)^{-1}(V)$ is $\delta$-open in $U_{i}$ for each $i \in I$. 
Since $U_{i}$ is $\delta$-open in $X$ for each $i \in I$. So by Lemma 2.5, $\left(f \mid U_{i}\right)^{-1}(V)$ is $\delta$-open in $X$ for each $i \in I$. But, $f^{-1}(V)=\mathrm{U}\left\{\left(f \mid U_{i}\right)^{-1}(V): i \in I\right\}$, then $f^{-1}(V) \in \tau_{\delta}$ because $\tau_{\delta}$ is a topology on $X$. This implies that $f$ is $\delta$-continuous.

\subsection{DELTA - IRRESOLUTE FUNCTIONS}

In this section, the functions to be considered are those for which inverses of $\delta$-open sets are $\delta$-open. We investigate some properties and characterizations of such functions.

Definition 2.7. Let $(X, \tau)$ and $\quad(Y, \sigma) \quad$ be topological spaces. A function $f:(X, \tau) \rightarrow(Y, \sigma)$ is called $\delta$-irresolute if the inverse image of each $\delta$-open set of $Y$ is a $\delta$-open set in $X$.

Theorem 2.8. Let $f:(X, \tau) \rightarrow(Y, \sigma)$ be a function between topological spaces. Then the following are equivalent:

(1) $f$ is $\delta$-irresolute;

(2) the inverse image of each $\delta$-closed set in $Y$ is a $\delta$-closed set in $X$;

(3) $C l_{\delta}\left[f^{-1}(V)\right] \subseteq f^{-1}\left[C l_{\delta}(V)\right]$ for every $V \subseteq Y$;

(4) $f\left[C l_{\delta}(U)\right] \subseteq C l_{\delta}[f(U)]$ for every $U \subseteq X$;

(5) $f^{-1}\left[\operatorname{Int}_{\delta}(B)\right] \subseteq \operatorname{Int}_{\delta}\left[f^{-1}(B)\right]$ for every $B \subseteq Y$.

Theorem 2.9. Prove that a function $f:(X, \tau) \rightarrow(Y, \sigma)$ is $\delta$-irresolute if and only if for each point $p$ in $X$ and each $\delta$-open set $B$ in $Y$ with $f(p) \in B$, there is a $\delta$-open set $A$ in $X$ such that $p \in A, f(A) \subseteq B$.
Proof. Necessity. Let $p \in X$ and $B \in \sigma_{\delta}$ such that $f(p) \in B$. Let $A=f^{-1}(B)$. Since $f$ is $\delta$-irresolute, $\quad A$ is $\delta$-open in $X$.Also $p \in f^{-1}(B)=A$ as $f(p) \in B$. Thus we have $f(A)=f\left[f^{-1}(B)\right] \subseteq B$.

Sufficiency. Let $B \in \sigma_{\delta}$, let $A=f^{-1}(B)$. We show that $A$ is $\delta$-open in $X$. For this let $x \in A$. It implies that $f(x) \in B$. Then by hypothesis, there exists $A_{x} \in \tau_{\delta}$ such that $x \in A_{x}$ and $f\left(A_{x}\right) \subseteq B$. Then $A_{x} \subseteq f^{-1}\left[f\left(A_{x}\right)\right] \subseteq f^{-1}(B)=A . \quad$ Thus $A=\mathrm{U}\left\{A_{x}: x \in A\right\}$. It follows that $A$ is $\delta$-open in $X$. Hence $f$ is $\delta$-irresolute.

Definition 2.10. Let $(X, \tau)$ be a topological space. Let $x \in X$ and $N \subseteq X$. We say that $N$ is a $\delta$-neighborhood of $x$ if there exists a $\delta$-open set $M$ of $X$ such that $x \in M \subseteq N$.

Theorem 2.11. Prove that a function $f:(X, \tau) \rightarrow(Y, \sigma)$ is $\delta$-irresolute if and only if for each $x$ in $X$, the inverse image of every $\delta$-neighborhood of $f(x)$, is a $\delta$-neighborhood of $x$.

Proof. Necessity. Let $x \in X$ and let $B$ be a $\delta$-neighborhood of $f(x)$. Then there exists $U \in \sigma_{\delta}$ such that $f(x) \in U \subseteq B$. This implies that $x \in f^{-1}(U) \subseteq f^{-1}(B)$. Since $f$ is $\delta$-irresolute, so $f^{-1}(U) \in \tau_{\delta}$. Hence $f^{-1}(B)$ is a $\delta$-neighborhood of $x$.

Sufficiency. Let $B \in \sigma_{\delta}$. Put $A=f^{-1}(B)$. Let $x \in A$. Then $f(x) \in B$. But then, $B$ being $\delta$-open set, is a $\delta$-neighborhood of $f(x)$. So by hypothesis, $A=f^{-1}(B)$ is a $\delta$-neighborhood of $x$. Hence by definition, there exists $A_{x} \in \tau_{\delta}$ such that $x \in A_{x} \subseteq A$. Thus 
$A=\mathrm{U}\left\{A_{x}: x \in A\right\}$. It follows that $A$ is a $\delta$-open set in $X$.Therefore $f$ is $\delta$-irresolute.

Theorem 2.12. Prove that a function $f:(X, \tau) \rightarrow(Y, \sigma)$ is $\delta$-irresolute if and only if for each $X$ in $X$. and each $\delta$-neighborhood $U$ of $f(x)$, there is a $\delta$-neighborhood $V$ of $x$ such that $f(V) \subseteq U$.

Proof. Necessity. Let $x \in X$ and let $U$ be a $\delta$-neighborhood of $f(x)$. Then there exists $O_{f(x)} \in \sigma_{\delta}$ such that $f(x) \in O_{f(x)} \subseteq U$. It follows that $x \in f^{-1}\left[O_{f(x)}\right] \subseteq f^{-1}(U)$. By hypothesis, $f^{-1}\left[O_{f(x)}\right] \in \tau_{\delta}$. Let $V=f^{-1}(U)$. Then it follows that $V$ is a $\delta$-neighborhood of $x$ and $f(V)=f\left[f^{-1}(U)\right] \subseteq U$.

Sufficiency. Let $B \in \sigma_{\delta}$. Put $O=f^{-1}(B)$. Let $x \in O$. Then $f(x) \in B$. Thus $B$ is a $\delta$-neighborhood of $f(x)$. So by hypothesis, there exists a $\delta$-neighborhood $V_{x}$ of $x$ such that $f\left(V_{x}\right) \subseteq B$. Thus it follows that $x \in V_{x} \subseteq f^{-1}\left[f\left(V_{x}\right)\right] \subseteq f^{-1}(B)=O$. Since $V_{x}$ is a $\delta$-neighborhood of $x$, so there exists an $O_{x} \in \tau_{\delta}$ such that $x \in O_{x} \subseteq V_{x}$. Hence $x \in O_{x} \subseteq O, O_{x} \in \tau_{\delta}$. Thus $O=\mathrm{U}\left\{O_{x}: x \in O\right\}$. It follows that $O$ is $\delta$-open in $X$. Therefore, $f$ is $\delta$-irresolute.

Theorem 2.13. Prove that a function $f:(X, \tau) \rightarrow(Y, \sigma)$ is $\delta$-irresolute if and only if $f\left[D_{\delta}(A)\right] \subseteq f(A) \mathrm{U} D_{\delta}[f(A)]$, for all $A \subseteq X$.

Proof. Necessity. Let $f:(X, \tau) \rightarrow(Y, \sigma)$ be $\delta$-irresolute. Let $A \subseteq X$, and $a_{0} \in D_{\delta}(A)$. Assume that $f\left(a_{0}\right) \notin f(A)$ and let $V$ denote a $\delta$-neighborhood of $f\left(a_{0}\right)$. Since $f$ is $\delta$-irresolute, so by Theorem 2.12, there exists a $\delta$-neighborhood $U$ of $a_{0}$ such that
$f(U) \subseteq V$. From $a_{0} \in D_{\delta}(A)$, it follows that $U$ I $A \neq \phi$; there exists, therefore, at least one element $a \in U$ I $A$ such that $f(a) \in f(A)$ and $f(a) \in f(V)$. Since $f\left(a_{0}\right) \notin f(A)$, we have $f(a) \neq f\left(a_{0}\right)$. Thus every $\delta$-neighborhood of $f\left(a_{0}\right)$ contains an element of $f(A)$ different from $f\left(a_{0}\right)$, consequently, $f\left(a_{0}\right) \in D_{\delta}[f(A)]$. This proves necessity of the condition.

Sufficiency. Assume that $f$ is not $\delta$-irresolute. Then by Theorem 2.12, there exists $a_{0} \in X$ and a $\delta$-neighborhood $V$ of $f\left(a_{0}\right)$ such that every $\delta$-neighborhood $U$ of $a_{0}$ contains at least one element $a \in U$ for which $f(a) \notin V$. Put $A=\{a \in X: f(a) \notin V\}$. Then $a_{0} \notin A$ since $f\left(a_{0}\right) \in V$, and therefore $f\left(a_{0}\right) \notin A$; also $f\left(a_{0}\right) \notin D_{\delta}[f(A)]$ since $V$ I $\left(V-\left\{f\left(a_{0}\right)\right\}\right)=\phi$. It follows that $f\left(a_{0}\right) \in f\left[D_{\delta}(A)\right]-\left[f(A) \cup D_{\delta}(f(A))\right] \neq \phi$, which is a contradiction to the given condition. The condition of the Theorem is therefore sufficient and the theorem is proved.

Theorem 2.14. Let $f:(X, \tau) \rightarrow(Y, \sigma)$ be a oneto-one function. Then $\mathrm{f}$ is $\delta$-irresolute if and only if $f\left[D_{\delta}(A)\right] \subseteq D_{\delta}[f(A)]$, for all $A \subseteq X$.

Proof. Necessity. Let $f$ be $\delta$-irresolute. Let $A \subseteq X, a_{0} \in D_{\delta}(A)$ and $V$ be a $\delta$-neighborhood of $f\left(a_{0}\right)$. Since $f$ is $\delta$-irresolute, so by Theorem 2.12, there exists a $\delta$-neighborhood $U$ of $a_{0}$ such that $f(U) \subseteq V$. But $a_{0} \in D_{\delta}(A)$; hence there exists an element $a \in U$ I $A$ such that $a \neq a_{0}$; then $f(a) \in f(A)$ and, since $f$ is one to one, $f(a) \neq f\left(a_{0}\right)$. Thus every $\delta$-neighborhood $V$ of $f\left(a_{0}\right)$ contains an element of $f(A)$ different from $f\left(a_{0}\right)$; consequently $f\left(a_{0}\right) \in D_{\delta}[f(A)]$. We have therefore $f\left[D_{\delta}(A)\right] \subseteq D_{\delta}[f(A)]$. 
Sufficiency. Follows from Theorem 2.13.

\subsection{DELTA - OPEN FUNCTIONS}

The purpose of this section is to investigate some characterizations of $\delta$-open mappings.

Definition 2.15. Let $(X, \tau)$ and $(Y, \sigma)$ be topological spaces. A function $f:(X, \tau) \rightarrow(Y, \sigma)$ is called $\delta$-open if for every open set $G$ in $X, f(G)$ is a $\delta$-open set in $Y$.

Theorem 2.16. Prove that a mapping $f:(X, \tau) \rightarrow(Y, \sigma)$ is $\delta$-open if and only if for each $x \in X$, and $U \in \tau$ such that $x \in U$, there exists a $\delta$-open set $W \subseteq Y$ containing $f(x)$ such that $W \subseteq f(U)$.

Proof. Follows immediately from Definition 2.15.

Theorem 2.17. Let $f:(X, \tau) \rightarrow(Y, \sigma)$ be $\delta$-open. If $W \subseteq Y$ and $F \subseteq X$ is a closed set containing $f^{-1}(W)$, then there exists a $\delta$-closed $H \subseteq Y$ containing $W$ such that $f^{-1}(H) \subseteq F$.

Proof. Let $H=Y-f(Y-F)$. Since $f^{-1}(W) \subseteq F$, we have $f^{-1}(Y-F) \subseteq(Y-W)$. Since $f$ is $\delta$-open, then $H$ is $\delta$-closed and $f^{-1}(H)=X-f^{-1}[f(X-F)] \subseteq X-(X-F)=F$.

Theorem 2.18. Let $f:(X, \tau) \rightarrow(Y, \sigma)$ be a $\delta$-open function and let $B \subseteq Y$. Then $f^{-1}\left[C l_{\delta}\left(\operatorname{Int}_{\delta}\left(C l_{\delta}(B)\right)\right)\right] \subseteq C l\left[f^{-1}(B)\right]$.

Proof. $\mathrm{Cl}\left[f^{-1}(B)\right] \quad$ is closed in $X$ containing $f^{-1}(B)$. By Theorem 2.17, there exists a $\delta$-closed set $B \subseteq H \subseteq Y$ such that $f^{-1}(H) \subseteq C l\left[f^{-1}(B)\right]$.

Thus,

$$
\begin{aligned}
& f^{-1}\left[C l_{\delta}\left(\operatorname{Int}_{\delta}\left(C l_{\delta}(B)\right)\right)\right] \subseteq f^{-1}\left[C l_{\delta}\left(\operatorname{Int}_{\delta}\left(C l_{\delta}(H)\right)\right)\right] \\
& \subseteq f^{-1}[H] \subseteq C l\left[f^{-1}(B)\right] .
\end{aligned}
$$

Theorem 2.19. Prove that a function $f:(X, \tau) \longrightarrow(Y, \sigma)$ is $\delta$-open if and only

$f[\operatorname{Int}(A)] \subseteq \operatorname{Int}_{\delta}[f(A)]$, for all $A \subseteq X$.

Proof.Necessity. Let $A \subseteq X$. Let $x \in \operatorname{Int}(A)$. Then there exists $U_{x} \in \tau$ such that $x \in U_{x} \subseteq A$. So $f(x) \in f\left(U_{x}\right) \subseteq f(A)$. and by hypothesis, $f\left(U_{x}\right) \in \sigma_{\delta}$. Hence

$$
\begin{aligned}
& f(x) \in \operatorname{Int}_{\delta}[f(A)] . \\
& f[\operatorname{Int}(A)] \subseteq \operatorname{Int}_{\delta}[f(A)] .
\end{aligned}
$$$$
\text { Thus }
$$

Sufficiency. Let $U \in \tau$. Then by hypothesis,

$f[\operatorname{Int}(U)] \subseteq \operatorname{Int}_{\delta}[f(U)]$. Since

$\operatorname{Int}(U)=U$ as $U$ is open. Also

Int $_{\delta}[f(U)] \subseteq f(U)$. Hence

$f(U)=\operatorname{Int}_{\delta}[f(U)]$. Thus $f(U)$ is

$\delta$-open open in $Y$. So $f$ is $\delta$-open.

Remark 2.20. The equality may not hold in the preceding Theorem.

Theorem 2.21. Prove that a function $f:(X, \tau) \rightarrow(Y, \sigma)$ is $\delta$-open if and only if $\operatorname{Int}\left[f^{-1}(B)\right] \subseteq f^{-1}\left[\operatorname{Int}_{\delta}(B)\right]$, for all $B \subseteq Y$.

Proof. Necessity. Let $B \subseteq Y$. Since $\operatorname{Int}\left[f^{-1}(B)\right]$ is open in $X$ and $f$ is $\delta$-open, $f\left[\operatorname{Int}\left(f^{-1}(B)\right)\right]$ is $\delta$-open in $Y$. Also we have $f\left[\operatorname{Int}\left(f^{-1}(B)\right)\right] \subseteq f\left[f^{-1}(B)\right] \subseteq B . \quad$ Hence, $f\left[\operatorname{Int}\left(f^{-1}(B)\right)\right] \subseteq \operatorname{Int}_{\delta}(B) . \quad$ Therefore $\operatorname{Int}\left(f^{-1}(B)\right) \subseteq f^{-1}\left[\operatorname{Int}_{\delta}(B)\right]$. 
Sufficiency. Let $A \subseteq X$. Then $f(A) \subseteq Y$. Hence by hypothesis, we obtain $\operatorname{Int}(A) \subseteq \operatorname{Int}\left[f^{-1}(f(A))\right] \subseteq f^{-1}\left[\operatorname{Int}_{\delta}(f(A))\right]$.

Thus $f[\operatorname{int}(A)] \subseteq \operatorname{Int}_{\delta}[f(A)]$, for all $A \subseteq X$. Hence, by Theorem 2.19, $f$ is $\delta$-open.

Theorem 2.22. Let $f:(X, \tau) \rightarrow(Y, \sigma)$ be a mapping. Then a necessary and sufficient condition for $f$ to be $\delta$-open is that $f^{-1}\left[C l_{\delta}(B)\right] \subseteq C l\left[f^{-1}(B)\right]$ for every subset $B$ of $Y$.

Proof. Necessity. Assume $f$ is $\delta$-open. Let $B \subseteq Y . \quad$ Let $\quad x \in f^{-1}\left[C l_{\delta}(B)\right] . \quad$ Then $f(x) \in C l_{\delta}(B)$. Let $U \in \tau$ such that $x \in U$. Since $f$ is $\delta$-open, then $f(U)$ is a $\delta$-open set in $Y$. Therefore, $\quad B \mathrm{I} f(U) \neq \phi$. Then $U$ I $f^{-1}(B) \neq \phi$. Hence $\quad x \in C l\left[f^{-1}(B)\right] . \quad$ We conclude that $f^{-1}\left[C l_{\delta}(B)\right] \subseteq C l\left[f^{-1}(B)\right]$.

Sufficiency. Let $B \subseteq Y$. Then $(Y-B) \subseteq Y$. By hypothesis, $\quad f^{-1}\left[C l_{\delta}(Y-B)\right] \subseteq C l\left[f^{-1}(Y-B)\right]$. This implies that $X-C l\left[f^{-1}(Y-B)\right] \subseteq X-f^{-1}\left[C l_{\delta}(Y-B)\right]$.

Hence

$X-C l\left[X-f^{-1}(B)\right] \subseteq f^{-1}\left[Y-C l_{\delta}(Y-B)\right] . \quad$ By applying Theorem 10[18], $\operatorname{Int}\left[f^{-1}(B)\right] \subseteq f^{-1}\left[\operatorname{Int}_{\delta}(B)\right] . \quad$ Now $\quad$ form Theorem 2.21, it follows that $f$ is $\delta$-open.

\subsection{DELTA - CLOSED FUNCTIONS}

In this section we introduce $\delta$-closed functions and study certain properties and characterizations of this type of functions.

Definition 2.23. A mapping $f:(X, \tau) \rightarrow(Y, \sigma)$ is called $\delta$-closed if the image of each closed set in $X$ is a $\delta$-closed set in $Y$.
Theorem 2.24. Prove that a mapping $f:(X, \tau) \rightarrow(Y, \sigma)$ is $\delta$-closed if and only if $C l_{\delta}[f(A)] \subseteq f[C l(A)]$ for each $A \subseteq X$.

Proof. Necessity. Let $f$ be $\delta$-closed and let $A \subseteq X$. Then $f(A) \subseteq f[C l(A)]$ and $f[C l(A)]$ is a $\delta$-closed set in $Y$. Thus

$$
C l_{\delta}[f(A)] \subseteq f[C l(A)] .
$$

Sufficiency. Suppose that $C l_{\delta}[f(A)] \subseteq f[C l(A)]$, for each $A \subseteq X$. Let $A \subseteq X$ be a closed set. Then $C l_{\delta}[f(A)] \subseteq f[C l(A)]=f(A)$. This shows that $f(A)$ is a $\delta$-closed set. Hence $f$ is $\delta$-closed.

Theorem 2.25. Let $f:(X, \tau) \rightarrow(Y, \sigma)$ be $\delta$-closed. If $V \subseteq Y$ and $E \subseteq X$ is an open set containing $f^{-1}(V)$, then there exists a $\delta$-open set $G \subseteq Y$ containing $V$ such that $f^{-1}(G) \subseteq E$.

Proof. Let $G=Y-f(X-E)$. Since $f^{-1}(V) \subseteq E$, we have $f(X-E) \subseteq Y-V$. Since $f$ is $\delta$-closed, then $G$ is a $\delta$-open set

and $f^{-1}(G)=X-f^{-1}[f(X-E)] \subseteq X-(X-E)=E$.

Theorem 2.26. Suppose that $f:(X, \tau) \rightarrow(Y, \sigma)$ is a $\delta$-closed mapping. Then $\operatorname{Int}_{\delta}\left[C_{\delta}(f(A))\right] \subseteq f[C l(A)]$ for every subset $A$ of $X$.

Proof. Suppose $f$ is a $\delta$-closed mapping and $A$ is an arbitrary subset of $X$. Then $f[C l(A)]$ is $\delta$-closed in $Y$. Then Int $_{\delta}\left[C l_{\delta}(f(C l(A)))\right] \subseteq f[C l(A)]$. But also $\operatorname{Int}_{\delta}\left[C l_{\delta}(f(A))\right] \subseteq \operatorname{Int}_{\delta}\left[C l_{\delta}(f(C l(A)))\right]$. Hence $\operatorname{Int}_{\delta}\left[C l_{\delta}(f(A))\right] \subseteq f[C l(A)]$.

Theorem 2.27. Let $f:(X, \tau) \rightarrow(Y, \sigma)$ be a $\delta$-closed function, and $B, C \subseteq Y$. 
Proof. (1) If $U$ is an open neighborhood of $f^{-1}(B)$, then there exists a $\delta$-open neighborhood $\quad V$ of $B$ such that $f^{-1}(B) \subseteq f^{-1}(V) \subseteq U$.

(2) If $f$ is also onto, then if $f^{-1}(B)$ and $f^{-1}(C)$ have disjoint open neighborhoods, so have $B$ and $C$.

Proof. (1) Let $\quad V=Y-f(X-U)$. Then $V^{c}=Y-V=f\left(U^{c}\right)$. Since $f$ is $\delta$-closed, so $V$ is a $\delta$-open set. Since $f^{-1}(B) \subseteq U$, we have $V^{c}=f\left(U^{c}\right) \subseteq f\left[f^{-1}\left(B^{c}\right)\right] \subseteq B^{c}$. Hence, $B \subseteq V$, and thus $V$ is a $\delta$-open neighborhood of $B$. Further $U^{c} \subseteq f^{-1}\left[f\left(U^{c}\right)\right]=f^{-1}\left(V^{c}\right)=\left[f^{-1}(V)\right]^{c}$. This proves that $f^{-1}(V) \subseteq U$.

(2) If $f^{-1}(B)$ and $f^{-1}(C)$ have disjoint open neighborhoods $M$ and $N$, then by (1), we have $\delta$-open neighborhoods $U$ and $V$ of $B$ and $C$ respectively such that $f^{-1}(B) \subseteq f^{-1}(U) \subseteq \operatorname{Int}_{\delta}(M)$ and $f^{-1}(C) \subseteq f^{-1}(V) \subseteq \operatorname{Int}_{\delta}(N)$. Since $M$ and $N$ are disjoint, so are $\operatorname{Int}_{\delta}(M)$ and $\operatorname{Int}_{\delta}(N)$, hence so $f^{-1}(U)$ and $f^{-1}(V)$ are disjoint as well. It follows that $U$ and $V$ are disjoint too as $f$ is onto.

Theorem 2.28. Prove that a surjective mapping $f:(X, \tau) \rightarrow(Y, \sigma)$ is $\delta$-closed, if and only if for each subset $B$ of $Y$ and each open set $U$ in $X$ containing $f^{-1}(B)$, there exists a $\delta$-open set $V$ in $Y$ containing $B$ such that $f^{-1}(V) \subseteq U$.

Proof. Necessity. This follows from (1) of Theorem 2.27.

Sufficiency. Suppose $F$ is an arbitrary closed set in $X$. Let $y$ be an arbitrary point in $Y-f(F)$.

Then $f^{-1}(y) \subseteq X-f^{-1}[f(F)] \subseteq(X-F)$ and $(X-F)$ is open in $X$. Hence by hypothesis, there exists a $\delta$-open set $V_{y}$ containing $y$ such that $f^{-1}\left(V_{y}\right) \subseteq(X-F)$. This implies that $y \in V_{y} \subseteq[Y-f(F)] . \quad$ Thus $Y-f(F)=\mathrm{U}\left\{V_{y}: y \in Y-f(F)\right\}$. Hence $Y-f(F)$, being a union of $\delta$-open sets, is $\delta$-open. Thus its complement $f(F)$ is $\delta$-closed. This shows that $f$ is $\delta$-closed.

Theorem 2.29. Let $f:(X, \tau) \rightarrow(Y, \sigma)$ be a bijection. Then the following are equivalent:

(a) $f$ is $\delta$-closed.

(b) $f$ is $\delta$-open.

(c) $f^{-1}$ is $\delta$-continuous.

Proof. $(\mathrm{a}) \Rightarrow(\mathrm{b}): \quad$ Let $U \in \tau$. Then $X-U$ is closed in $X$. By $(\mathrm{a}), f(X-U)$ is $\delta$-closed in $Y$. But $f(X-U)=f(X)-f(U)=Y-f(U)$. Thus $f(U)$ is $\delta$-open in $Y$. This shows that $f$ is $\delta$-open.

(b) $\Rightarrow(\mathrm{c})$ : Let $U \subseteq X$. be an open set. Since $f$ is $\delta$-open. So $f(U)=\left(f^{-1}\right)^{-1}(U)$ is $\delta$-open in $Y$. Hence $f^{-1}$ is $\delta$-continuous.

(c) $\Rightarrow(a)$ : Let $A$ be an arbitrary closed set in $X$. Then $X-A$ is open in $X$. Since $f^{-1}$ is $\delta$-continuous, $\left(f^{-1}\right)^{-1}(X-A)$ is $\delta$-open in $Y$. $\operatorname{But}\left(f^{-1}\right)^{-1}(X-A)=f(X-A)=Y-f(A)$. Thus $f(A)$ is $\delta$-closed in $Y$. This shows that $f$ is $\delta$-closed.

Remark 2.30. A bijection $f:(X, \tau) \rightarrow(Y, \sigma)$ may be open and closed but neither $\delta$-open nor $\delta$-closed.

\subsection{PRE-DELTA - OPEN FUNCTIONS}


The purpose of this section is to introduce and discuss certain properties and characterizations of pre- $\delta$-open functions.

Definition 2.31. Let $(X, \tau)$ and $(Y, \sigma)$ be topological spaces. Then a function $f:(X, \tau) \rightarrow(Y, \sigma)$ is said to be pre- $\delta$-open if and only if for each $A \in \tau_{\delta}, f(A) \in \sigma_{\delta}$.

Theorem 2.32. Let $f:(X, \tau) \rightarrow(Y, \sigma) \quad$ and $g:(Y, \sigma) \rightarrow(Z, \mu)$ be any two pre- $\delta$-open functions. Then the composition function $g \circ f:(X, \tau) \rightarrow(Z, \mu)$ is a pre- $\delta-$ open function.

Proof. Let $U \in \tau_{\delta}$. Then $f(U) \in \sigma_{\delta}$. Since $f$ is pre- $\delta$-open. But then $g(f(U)) \in \mu_{\delta}$ as $g$ is pre- $\delta-$ open. Hence, $g \circ f$ is pre- $\delta-$ open.

Theorem 2.33. Prove that a mapping $f:(X, \tau) \rightarrow(Y, \sigma)$ is pre- $\delta$-open if and only if for each $x \in X$ and for any $U \in \tau_{\delta}$ such that $x \in U$, there exists $V \in \sigma_{\delta}$ such that $f(x) \in V$ and $V \subseteq f(U)$.

Proof. Routine.

Theorem 2.34. Prove that a mapping $f:(X, \tau) \rightarrow(Y, \sigma)$ is pre- $\delta-$ open if and only if for each $x \in X$ and for any $\delta$-neighborhood $\mathrm{U}$ of $x$ in $X$, there exists a $\delta$-neighborhood $V$ of $f(x)$ in $Y$ such that $V \subseteq f(U)$.

Proof. Necessity. Let $x \in X$ and let $U$ be a $\delta$-neighborhood of $x$. Then there exists $W \in \tau_{\delta} \quad$ such that $\quad x \in W \subseteq U . \quad$ Then $f(x) \in f(W) \subseteq f(U)$. But $f(W) \in \sigma_{\delta}$ as $f$ is pre- $\delta$-open. Hence $V=f(W)$ is a $\delta$-neighborhood of $f(x)$ and $V \subseteq f(U)$.

Sufficiency. Let $U \in \tau_{\delta}$. Let $x \in U$. Then $U$ is a $\delta$-neighborhood of $x$. So by hypothesis, there exists a $\delta$-neighborhood $V_{f(x)}$ of $f(x)$ such that $f(x) \in V_{f(x)} \subseteq f(U)$. It follows at once that
$f(U)$ is a $\delta$-neighborhood of each of its points. Therefore $f(U)$ is $\delta$-open. Hence $f$ is pre- $\delta$-open.

Theorem 2.35. Prove that a function $f:(X, \tau) \rightarrow(Y, \sigma)$ is pre- $\delta-$ open if and only if $f\left[\operatorname{Int}_{\delta}(A)\right] \subseteq \operatorname{Int}_{\delta}[f(A)]$, for all $A \subseteq X$.

Proof. Necessity. Let $A \subseteq X$. Let $x \in \operatorname{Int}_{\delta}(A)$. Then there exists $U_{x} \in \tau_{\delta}$ such that $x \in U_{x} \subseteq A$. So $f(x) \in f\left(U_{x}\right) \subseteq f(A)$ and by hypothesis, $f\left(U_{x}\right) \in \sigma_{\delta}$. Hence $f(x) \in \operatorname{Int}_{\delta}[f(A)]$. Thus $f\left[\operatorname{Int}_{\delta}(A)\right] \subseteq \operatorname{Int}_{\delta}[f(A)]$.

Sufficiency. Let $U \in \tau_{\delta}$. Then by hypothesis, $f\left[\operatorname{Int}_{\delta}(U)\right] \subseteq \operatorname{Int}_{\delta}[f(U)]$. Since $\operatorname{Int}_{\delta}(U)=U$ as $U$ is $\delta$-open. Also $\operatorname{Int}_{\delta}[f(U)] \subseteq f(U)$. Hence $f(U)=\operatorname{Int}_{\delta}[f(U)]$. Thus $f(U)$ is $\delta$-open in $Y$. So $f$ is pre- $\delta-o p e n$.

We remark that the equality does not hold in Theorem 2.35 as the following example shows.

Example 2.36. Let $X=Y=\{1,2\}$. suppose $X$ is antidiscrete and $Y$ is discrete. Let $f=I d$., $A=\{1\}$. Then $\phi=f\left[\operatorname{Int}_{\delta}(A)\right] \neq \operatorname{Int}_{\delta}[f(A)]=\{1\}$.

Theorem 2.37. Prove that a function $f:(X, \tau) \rightarrow(Y, \sigma)$ is pre- $\delta-$ open if and only if $\operatorname{Int}_{\delta}\left[f^{-1}(B)\right] \subseteq f^{-1}\left[\operatorname{Int}_{\delta}(B)\right]$, for all $B \subseteq Y$.

Proof. Necessity. Let $B \subseteq Y$. Since Int $\left[f^{-1}(B)\right]$ is $\delta$-open in $X$ and $f$ is pre- $\delta$-open, $f\left[\operatorname{Int}_{\delta}\left(f^{-1}(B)\right)\right]$ is $\delta$-open in $Y$. Also we have $f\left[\operatorname{Int}_{\delta}\left(f^{-1}(B)\right)\right] \subseteq f\left[f^{-1}(B)\right]$ $\subseteq B . \quad$ Hence, $\quad f\left[\operatorname{Int}_{\delta}\left(f^{-1}(B)\right)\right] \subseteq \operatorname{Int}_{\delta}(B)$. Therefore $\operatorname{Int}_{\delta}\left[f^{-1}(B)\right] \subseteq f^{-1}\left[\operatorname{Int}_{\delta}(B)\right]$.

Sufficiency. Let $A \subseteq X$. Then $f(A) \subseteq Y$. Hence by hypothesis, we obtain $\operatorname{Int}_{\delta}(A) \subseteq \operatorname{Int}_{\delta}\left[f^{-1}(f(A))\right] \subseteq f^{-1}\left[\operatorname{Int}_{\delta}(f(A))\right]$. 
This implies $f\left[\operatorname{Int}_{\delta}(A)\right] \subseteq f\left[f^{-1}\left(\operatorname{Int}_{\delta}(f(A))\right)\right] \subseteq \operatorname{Int}_{\delta}[f(A)]$. Thus $f\left[\operatorname{Int}_{\delta}(A)\right] \subseteq \operatorname{Int}_{\delta}[f(A)]$, for all $A \subseteq X$. Hence, by Theorem 2.35, $f$ is pre- $\delta$-open.

Theorem 2.38. Prove that a mapping $f:(X, \tau) \rightarrow(Y, \sigma)$ is pre- $\delta-$ open if and only if $f^{-1}\left[C l_{\delta}(B)\right] \subseteq C l_{\delta}\left[f^{-1}(B)\right]$, for every subset $B$ of $Y$.

Proof. Necessity. Let $B \subseteq Y$. Let $x \in f^{-1}\left[C l_{\delta}(B)\right]$. Then $f(x) \in C l_{\delta}(B)$. Let $U \in \tau_{\delta} \quad$ such that $x \in U$. By hypothesis, $f(U) \in \sigma_{\delta} \quad$ and $\quad f(x) \in f(U) . \quad$ Thus $f(U) \mathrm{I} B \neq \phi$. Hence $U$ I $f^{-1}(B) \neq \phi$. Therefore, $x \in C l_{\delta}\left[f^{-1}(B)\right], \quad$ So $\quad$ we obtain $f^{-1}\left[C l_{\delta}(B)\right] \subseteq C l_{\delta}\left[f^{-1}(B)\right]$.

Sufficiency. Let $B \subseteq Y$. Then $(Y-B) \subseteq Y$. By hypothesis, $\quad f^{-1}\left[C l_{\delta}(Y-B)\right] \subseteq C l_{\delta}\left[f^{-1}(Y-B)\right]$. This implies that $X-C l_{\delta}\left[f^{-1}(Y-B)\right] \subseteq X-f^{-1}\left[C l_{\delta}(Y-B)\right]$.

Hence $X-C l_{\delta}\left[X-f^{-1}(B)\right] \subseteq f^{-1}\left[Y-C l_{\delta}(Y-B)\right] . \quad$ By Theorem 2.7(6)[20], $\operatorname{Int}_{\delta}\left[f^{-1}(B)\right] \subseteq f^{-1}\left[\operatorname{Int}_{\delta}(B)\right]$. Now by Theorem 2.37, it follows that $f$ is pre- $\delta-$ open.

Theorem 2.39. Let $f:(X, \tau) \rightarrow(Y, \sigma) \quad$ and $g:(Y, \sigma) \rightarrow(Z, \mu)$ be two mappings such that $g \circ f:(X, \tau) \rightarrow(Z, \mu)$ is $\delta$-irresolute. Then

(1) If $g$ is a pre- $\delta$-open injection, then $f$ is $\delta$-irresolute.

(2) If $f$ is a pre- $\delta$-open surjection, then $g$ is $\delta$-irresolute.

Proof. (1) Let $U \in \sigma_{\delta}$. Then $g(U) \in \mu_{\delta}$ since $g$ is pre- $\delta$-open. Also $g$ of is $\delta$-irresolute. Therefore, we have $(g \circ f)^{-1}[g(U)] \in \tau_{\delta}$. Since $g$ is an injection, so we have : $(g \circ f)^{-1}[g(U)]=\left(f^{-1} \circ g^{-1}\right)[g(U)]=$ $f^{-1}\left[g^{-1}(g(U))\right]=f^{-1}(U)$. Consequently $f^{-1}(U)$ is $\delta$-open in $X$. This proves that $f$ is $\delta$-irresolute.

(2) Let $V \in \mu_{\delta}$. Then $(g \circ f)^{-1}(V) \in \tau_{\delta}$ since $g \circ f$ is $\delta$-irresolute. Also $f$ is pre- $\delta$-open, $f\left[(g \circ f)^{-1}(V)\right]$ is $\delta$-open in $Y$. Since $f$ is surjective, we note that $f\left[(g \circ f)^{-1}(V)\right]=$ $\left[f \circ(g \circ f)^{-1}\right](V)=\left[f \circ\left(f^{-1} \circ g^{-1}\right)\right](V)=\left[\left(f \circ f^{-1}\right) \circ g^{-1}(V)\right]=g^{-1}(V)$ Hence $g$ is $\delta$-irresolute.

\subsection{PRE - DELTA - CLOSED FUNCTIONS}

In this last section, we introduce and explore several properties and characterizations of pre $-\delta$-closed functions.

Definition 2.40. A function $f:(X, \tau) \rightarrow(Y, \sigma)$ is said to be pre- $\delta$-closed if and only if the image set $f(A)$ is $\delta$-closed for each $\delta$-closed subset $A$ of $X$.

Theorem 2.41. The composition of two pre $-\delta$-closed mappings is a pre $-\delta-$ closed mapping.

Pr oof. The straight forward proof is omitted.

Theorem 2.42. Prove that a mapping $f:(X, \tau) \rightarrow(Y, \sigma)$ is pre- $\delta$-closed if and only if $C l_{\delta}[f(A)] \subseteq f\left[C l_{\delta}(A)\right]$ for every subset $A$ of $X$.

Proof. Necessity. Suppose $f$ is a pre- $\delta$-closed mapping and $A$ is an arbitrary subset of $X$. Then $f\left[C l_{\delta}(A)\right]$ is $\delta$-closed in $Y$. Since $f(A) \subseteq f\left[C l_{\delta}(A)\right]$, we obtain $C l_{\delta}[f(A)] \subseteq f\left[C l_{\delta}(A)\right]$. 
Sufficiency. Suppose $F$ is an arbitrary $\delta$-closed set in $X$. By hypothesis, we obtain $f(F) \subseteq C l_{\delta}[f(F)] \subseteq f\left[C l_{\delta}(F)\right]=f(F)$. Hence $f(F)=C l_{\delta}[f(F)]$. Thus $f(F)$ is $\delta$-closed in $Y$. It follows that $f$ is pre- $\delta-$ closed.

Theorem 2.43. Let $f:(X, \tau) \rightarrow(Y, \sigma)$ be a pre $-\delta$-closed function, and $B, C \subseteq Y$.

(1) If $U$ is a $\delta$-open neighborhood of $f^{-1}(B)$, then there exists a $\delta$-open neighborhood $V$ of $B$ such that $f^{-1}(B) \subseteq f^{-1}(V) \subseteq U$.

(2) If $f$ is also onto, then if $f^{-1}(B)$ and $f^{-1}(C)$ have disjoint $\delta$-open neighborhoods, so have $B$ and $C$.

Proof. (1) Let $V=Y-f(X-U)$. Then $V^{c}=Y-V=f\left(U^{c}\right)$. Since $f$ is pre- $\delta-$ closed, so $V$ is $\delta$-open. Since $f^{-1}(B) \subseteq U$, we have $V^{c}=f\left(U^{c}\right) \subseteq f\left[f^{-1}\left(B^{c}\right)\right] \subseteq B^{c}$. Hence, $B \subseteq V$, and thus $V$ is a $\delta$-open neighborhood of $B$. Further

$U^{c} \subseteq f^{-1}\left[f\left(U^{c}\right)\right]=f^{-1}\left(V^{c}\right)=\left[f^{-1}(V)\right]^{c}$.

This proves that $f^{-1}(V) \subseteq U$.

(2) If $f^{-1}(B)$ and $f^{-1}(C)$ have disjoint $\delta$-open neighborhoods $M$ and $N$, then by (1), we have $\delta$-open neighborhoods $U$ and $V$ of $B$ and $C$ respectively such that $f^{-1}(B) \subseteq f^{-1}(U) \subseteq \operatorname{Int}_{\delta}(M) \quad$ and $f^{-1}(C) \subseteq f^{-1}(V) \subseteq \operatorname{Int}_{\delta}(N)$. Since $M$ and $N$ are disjoint, so are $\operatorname{Int}_{\delta}(M)$ and $\operatorname{Int}_{\delta}(N)$, and hence so $f^{-1}(U)$ and $f^{-1}(V)$ are disjoint as well. It follows that $U$ and $V$ are disjoint too as $f$ is onto.

Theorem 2.44. Prove that a surjective mapping $f:(X, \tau) \longrightarrow(Y, \sigma) \quad$ is pre $-\delta-$ closed if and only if for each subset $B$ of $Y$ and each $\delta$-open set $\mathrm{U}$ in $\mathrm{X}$ containing $f^{-1}(B)$, there exists a $\delta$-open set $V$ in Y containing $B$ such that $f^{-1}(V) \subseteq U$.

Proof.Necessity. This follows from (1) of Theorem 2.43.

Sufficiency. Suppose $F$ is an arbitrary $\delta-$ closed set in $X$. Let $y$ be an arbitrary point in $\quad Y-f(F)$. Then $f^{-1}(y) \subseteq X-f^{-1}[f(F)] \subseteq(X-F) \quad$ and $(X-F)$ is $\delta$-open in $\mathrm{X}$. Hence by hypothesis, there exists a $\delta$-open set $V_{y}$ containing $y$ such that $f^{-1}\left(V_{y}\right) \subseteq(X-F)$. This implies that $y \in V_{y} \subseteq[Y-f(F)]$. Thus $Y-f(F)=\mathrm{U}\left\{V_{y} \mid y \in Y-f(F)\right\}$. Hence $Y-f(F)$, being a union of $\delta$-open sets is $\delta$-open. Thus its complement $f(F)$ is $\delta$-closed. This shows that $f$ is $\delta$-closed.

Theorem 2.45. Let $f:(X, \tau) \longrightarrow(Y, \sigma)$ be a bijection. Then the following are equivalent:

(1) $f$ is pre $-\delta-$ closed.

(2) $f$ is pre $-\delta-o p e n$.

(3) $f^{-1}$ is $\delta$-irresolute.

Proof. (1) $\Rightarrow(2)$ : Let $U \in \tau_{\delta}$. Then $X-U$ is $\delta$-closed in $X$. By (1), $f(X-U)$ is $\delta$-closed in $Y$. But $f(X-U)=f(X)-f(U)=Y-f(U)$.

Thus $f(U)$ is $\delta$-open in $Y$. This shows that $f$ is pre- $\delta-$ open. 
$(2) \Rightarrow(3)$ : Let $\quad A \subseteq X$. Since $f$ is pre- $\delta$-open, so by Theorem 2.38, $f^{-1}\left[C l_{\delta}(f(A))\right] \subseteq C l_{\delta}\left[f^{-1}(f(A))\right] . \quad$ It implies that $C l_{\delta}[f(A)] \subseteq f\left[C l_{\delta}(A)\right]$.

Thus $\quad C l_{\delta}\left[\left(f^{-1}\right)^{-1}(A)\right] \subseteq\left(f^{-1}\right)^{-1}\left[C l_{\delta}(A)\right]$, for all $A \subseteq X$. Then by Theorem 2.8, it follows that $f^{-1}$ is $\delta$-irresolute.

$(3) \Rightarrow(1)$ : Let $A$ be an arbitrary $\delta$-closed set in $X$. Then $X-A$ is $\delta$-open in $X$. Since $f^{-1}$ is $\delta$-irresolute, $\left(f^{-1}\right)^{-1}(X-A)$ is $\delta$-open in $Y$. But $\left(f^{-1}\right)^{-1}(X-A)=f(X-A)=Y-f(A)$.

Thus $f(A)$ is $\delta$-closed in $Y$. This shows that $f$ is pre $-\delta-$ closed.

\section{ACKNOWLEDGEMENT}

The author is highly and gratefully indebted to the Prince Mohammad Bin Fahd University, Al Khobar, Saudi Arabia, for providing all necessary research facilities during the preparation of this research paper.

\section{REFERENCES}

[1] S.P. Arya and M. Deb, On $\theta$-continuous mappings, Math. Student 42(1974), 81-89.

[2] C.W. Baker, On $\theta-C$ open sets, Int. J. Math. Math. Sci. 15(1992), no. 2, 255-259.

[3] M. Caldas, D.N. Georgian, S. Jafari and T. Noiri, More on $\delta$-semiopen sets, Note di Mathematica, 22(2) (2003/2004), 113-126.

[4] J. Cao, M. Ganster, I. Reilly and M. Steiner, $\delta$-closure, $\theta$-closure, and generalized closed sets, Applied General Topology, 6(1) (2005), 79-86.

[5] R.F. Dickman, Jr. and J.R. Porter, $\theta$-closed subsets of Hausdorff spaces, Pacific J. Math. 59(1975), 407-415.

[6] R.F. Dickman, Jr. and J.R. Porter, $\theta$-perfect and $\theta$-absolutely closed functions, Illinois J. Math. 21(1977), 42-60.
[7] J. Dontchev, H. Maki, Groups of $\theta$ generalized homeomorphisms and the digital line, Topology and its Applications, 20(1998), 116.

[8] J. Dontchev and H. Maki, On $\theta$-generalized closed sets, Int. J. Math. Math. Sci. 22(1999), no. 2, 239-249.

[9] E. Ekici, ( $\delta$-pre,s)-continuous functions, Bulletin of the Malaysian Mathematical Sciences Society, Second Series 27(2004), no. 2, 237-251.

[10] E. Ekici, On $\delta$-semiopen sets and a generalization of functions, Bol. Soc. Mat. (38) vol. 23 (1-2) (2005), 73-84.

[11] Ganster1988 : M. Ganster, T. Noiri, I.L. Reilly, Weak and strong forms of $\theta$-irresolute functions, J. Inst. Math. Comput. Sci. 1(1) (1988), 19-29.

[12] S. Jafari, Some properties of $\theta$-continuous functions, Far East J. Math. Sci. 6(5) (1998), 689-696.

[13] D.S. Jankovic, On some separation axioms and $\theta$-closure, Mat. Vesnik 32(4) (1980), 439449.

[14] D.S. Jankovic, $\theta$-regular spaces, Internat. J. Math. \& Math. Sci. 8(1986), 515-619.

[15] J.E. Joseph, $\theta$-closure and $\theta$-subclosed graphs, Math., Chronicle 8(1979), 99-117.

[16] A. Kilicman, Z. Salleh, Some results on $(\delta-$ pre,s)-continuous functions, International Journal Math. Mat. Sci. 2006(2006), 1-11.

[17] M.M. Kovar, On $\theta$-regular spaces, Internat. J. Math. \& Math. Sci. 17(1994), 687-692.

[18] R.M. Latif, On characterizations of mappings, Soochow Journal of Mathematics, 19(4) (1993), 475-495.

[19] R.M. Latif, Semi-convergence of filters and nets, Mathematical Journal of Okayama University, 41(1999), 103-109.

[20] R.M. Latif, Characterizations and applications of $\gamma$-open sets, Soochow Journal of Mathematics, 32(3) (2006), 369-378.

[21] N. Levine, Semi-open sets and semicontinuity in topological space, Amer. Math. Monthly 70 (1963), 36-41. 
[22] P.E. Long, L.L. Herrington, The $\tau_{\theta}$ topology and faintly continuous functions, Kyungpook Math. J. 22(1982), 7-14.

[23] T. Noiri, On $\delta$-continuous functions, J. Korean Math. Soc., 16 (1980), 161-166.

[24] T. Noiri, S. Jafari, Properties of $(\theta, s)$ continuous functions, Topology and its Applications, 123(1) (2002), 167-179.

[25] J.H. Park, B. Y. Lee and M.J. Son, On $\delta$ semiopen sets in topological space, J. Indian Acad. Math., 19(1), (1997), 59-67.

[26] S. Raychaudhuri and M.N. Mukherjee, On $\delta$-almost continuity and $\delta$-preopen sets, Bulletin of the Institute of Mathematics, Academia Sinica 21 (1993), no. 4, 357-366.

[27] M. Saleh, Some applications of $\delta$-sets to $\mathrm{H}$ closed spaces, Q\&A Topology 17(1999), 203211.

[28] M. Saleh, On super and $\delta$-continuities, Mathematics and Mathematics Education, World Scientific, 2002, 281-291.

[29] M. Saleh, On $\theta$-closed sets and some forms of continuity, Archivum Mathematicum (BRNO) 40(2004), 383-393.

[30] N.V. Velicko, H-closed topological spaces, Mat. Sb., 98-112; English transl. (2), in Amer. Math. Soc. Transl., 78(1968), 102-118.

[31] S. Willard, General Topology, AddisonWesley Publishing Company, Inc., 1970.
Creative Commons Attribution License 4.0 (Attribution 4.0 International, CC BY 4.0)

This article is published under the terms of the Creative Commons Attribution License 4.0

https://creativecommons.org/licenses/by/4.0/deed.en_US 